Supporting Information for:

\title{
Experimental and Computational Study of Steric and Electronic Effects on the Coordination of Bulky, Water-Soluble Alkylphosphines to Palladium under Reducing Conditions: Correlation to Catalytic Activity
}

Rebecca B. DeVasher, Jason M. Spruell, David A. Dixon, Grant A. Broker, Robin D. Rogers, and Kevin H. Shaughnessy*

Department of Chemistry and Center for Green Manufacturing, The University of Alabama, Box 870336, Tuscaloosa, AL 35487-0336

Table of Contents

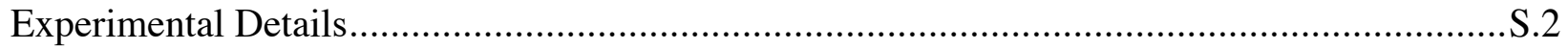

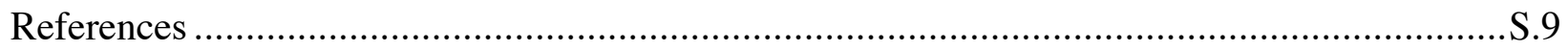

X-Ray Crystallographic Data ..................................................................................

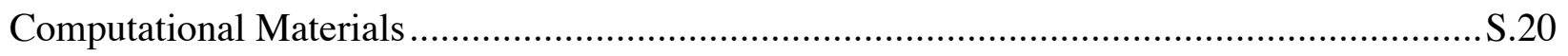

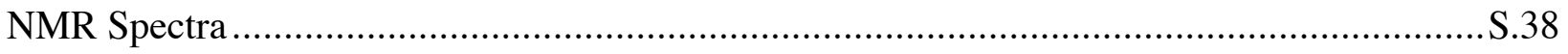




\section{Experimental Details}

General. Ligand syntheses were carried out under nitrogen using a dry box and Schlenk techniques unless noted. Coupling reactions were assembled in a drybox in screw-cap vials with a silicone/Teflon ${ }^{\circledR}$ septum or a round bottom sealed with a rubber septum. $\operatorname{Pd}(\mathrm{OAc})_{2}$, TPPTS (1), di-t-butylphosphine, and dicyclohexylphosphine were purchased from Strem and stored in a drybox under nitrogen. All boronic acids and aryl halides were purchased from Aldrich and were used without further purification. Di-t-phosphine-borane adduct, ${ }^{1} N$-methyl-4-piperidinyl tosylate $^{2}$ and Cy-Pip-phos ${ }^{2}$ were prepared by literature methods. THF and diethyl ether were freshly distilled from sodium-benzophenone ketyl under nitrogen prior to use. Anhydrous grade morpholine (Aldrich) was used as received and dispensed in a drybox. Water (deionized), acetonitrile, and methanol were degassed by sparging with nitrogen and/or exposure to vacuum.

A suitable crystal for X-ray characterization was mounted on a glass fiber and transferred to the goniometer. The crystal was cooled to $-100{ }^{\circ} \mathrm{C}$ in a stream of nitrogen gas and the data was collected on a Brüker SMART diffractometer with a CCD are detector, using graphice monochromated Mo-K $\alpha$ radiation. SHELXTL software, version 6.14, was used for solution and refinement. $^{3}$ Absorption corrections were made with SADABS. ${ }^{4}$ The structure was refined by full-matrix least-squares on $F^{2}$.

(2-Di-t-butylphospinoethyl)trimethylammonium chloride-borane adduct. Prepared by a modification of the synthesis reported by Grubbs. ${ }^{2}$ Di(t-butyl)phosphine-borane (1.588 g, 9.984 mmol) adduct was dissolved in $50 \mathrm{~mL}$ of THF under nitrogen and cooled to $-78{ }^{\circ} \mathrm{C}$. Butyl lithium (4.20 mL, $10.5 \mathrm{mmol}$ ) was added drop-wise to the phosphine solution. After the addition was complete, the reaction was stirred at $-78{ }^{\circ} \mathrm{C}$ for 15 minutes and then allowed to warm to room temperature and stirred for 2 hours. A second flask was charged with (2- 
chloroethyl)trimethylammonium chloride that had been finely ground in the drybox. The phosphine solution was cooled back to $-78{ }^{\circ} \mathrm{C}$ and added via cannula to the solid salt, which was also cooled to $-78{ }^{\circ} \mathrm{C}$. After the addition was complete, the reaction was allowed to warm to room temperature and then was refluxed for 24 hours to give a heavy white suspension. The THF was removed under reduced pressure in air and the solid residue was extracted with chloroform $(3 \times 100 \mathrm{~mL})$. The chloroform was removed under reduced pressure to give a colorless oil that was triturated with ether to give a slightly hygroscopic, colorless solid. The solid was dried in vacuo and stored in a dry box. Recovered (2.2126 g, $79 \%)$ of the borane adduct of $t$-Bu-Amphos. ${ }^{1} \mathrm{H}$ NMR $\left(\mathrm{D}_{2} \mathrm{O}, 360 \mathrm{MHz}\right): \quad \partial 3.70(\mathrm{dt}, J=4.47, J=13.56 \mathrm{~Hz}, 2 \mathrm{H}), 3.25$ (s, 9H), 2.30-2.22 (m, 2H), $1.35\left(\mathrm{~d},{ }^{3} J_{H-P}=13.56 \mathrm{~Hz}, 18 \mathrm{H}\right), 0.39$ (brq $(1: 1: 1: 1),{ }^{1} J_{H-B}=83.2 \mathrm{~Hz}$, 3H). ${ }^{31} \mathrm{P}$ NMR $\left(\mathrm{D}_{2} \mathrm{O}, 202.5 \mathrm{MHz}\right): \partial 43.0$ (brs).

(2-Di-t-butylphospinoethyl)trimethylammonium chloride ( $t$-Bu-Amphos). The phosphine-borane adduct $(2.093 \mathrm{~g}, 7.432 \mathrm{mmol})$ was taken up in $50 \mathrm{~mL}$ of degassed, dry morpholine under nitrogen and heated to $110{ }^{\circ} \mathrm{C}$. Upon heating, gas evolution was observed. After 3 hours, the reaction was allowed to cool to room temperature. The morpholine was removed in vacuo. The residue was dissolved in a minimum of degassed methanol and precipitated with ether $(50 \mathrm{~mL})$ to give a colorless crystalline solid, which was contaminated with morpholine-borane adduct. The crude material was placed in a sublimation apparatus and heated under vacuum (0.05 torr) at $110{ }^{\circ} \mathrm{C}$ for 2 hours. The non-volatile solid $(1.5892 \mathrm{~g}, 80 \%)$ recovered was pure by NMR spectroscopy. ${ }^{1} \mathrm{H} \mathrm{NMR}\left(\mathrm{CD}_{3} \mathrm{OD}, 360 \mathrm{MHz}\right): \delta 3.49$ (dt, $J=4.32$, $14.56 \mathrm{~Hz}, 2 \mathrm{H}), 3.18(\mathrm{~s}, 9 \mathrm{H}), 1.85(\mathrm{dt}, J=4.32,9.63 \mathrm{~Hz}, 2 \mathrm{H}), 1.19\left(\mathrm{~d},{ }^{3} J_{H-P}=11.71 \mathrm{~Hz}, 18 \mathrm{H}\right) .{ }^{13} \mathrm{C}$ $\operatorname{NMR}\left(\mathrm{CD}_{3} \mathrm{OD}, 90.6 \mathrm{MHz}\right): \delta 69.1\left(\mathrm{dt},{ }^{1} J_{\mathrm{C}-\mathrm{N}}=2.65 \mathrm{~Hz},{ }^{2} J_{\mathrm{C}-P}=49.09 \mathrm{~Hz}\right), 53.1\left(\mathrm{t},{ }^{1} J_{\mathrm{C}-\mathrm{N}}=3.98 \mathrm{~Hz}\right)$, $32.8\left(\mathrm{~d}, J_{C-P}=19.90 \mathrm{~Hz}\right), 30.0\left(\mathrm{~d}, J_{C-P}=13.26 \mathrm{~Hz}\right), 16.2\left(\mathrm{~d}, J_{C-P}=25.21 \mathrm{~Hz}\right) .{ }^{31} \mathrm{P} \mathrm{NMR}\left(\mathrm{D}_{2} \mathrm{O}, 202.5\right.$ 
$\mathrm{MHz}$ ): $\delta 22.3$ (s). Calculated for $\mathrm{C}_{13} \mathrm{H}_{31} \mathrm{ClNP}: \mathrm{C}, 58.30 ; \mathrm{H}, 11.67 ; \mathrm{N}, 5.23$. Found: C, 57.55; H, 11.33; N, 5.35. Carbon analysis was consistently low.

4-(Di-t-butylphospino)- $N$-methylpiperidine-borane adduct. Prepared by a modification of the procedure reported by Grubbs. ${ }^{2} \operatorname{Di}(t$-butyl)phosphine-borane adduct $(7.2062 \mathrm{~g}, 45.011$ $\mathrm{mmol})$ in THF (200 mL)was deprotonated with butyl lithium (19.0 mL, $47.5 \mathrm{mmol})$ as described for $t$-Bu-Amphos. This solution was cooled back to $-78{ }^{\circ} \mathrm{C}$ and a solution of freshly prepared $N$ methyl-4-piperidinyl tosylate $(11.54 \mathrm{~g}, 42.84 \mathrm{mmol})$ in $50 \mathrm{~mL}$ THF was added drop-wise via cannula. The resulting orange solution was allowed to warm to room temperature and then was refluxed overnight. The mixture was poured into $500 \mathrm{~mL}$ of saturated sodium bicarbonate and extracted with ether $(3 \times 150 \mathrm{~mL})$. The crude material was flash chromatographed on a silica gel column eluting with methanol to give $1.8105 \mathrm{~g}(16 \%)$ of $(N$-methylpiperidin-4-yl)di-tbutylphosphine-borane adduct. ${ }^{1} \mathrm{H}$ NMR $\left(\mathrm{CDCl}_{3}, 360 \mathrm{MHz}\right): \delta$ 3.05-3.00 (brm, 2H), 2.30-2.25 (brm, 2H), $2.30(\mathrm{~s}, 3 \mathrm{H}), 2.05-1.90(\mathrm{~m}, 4 \mathrm{H}), 1.85-1.70(\mathrm{~m}, 1 \mathrm{H}), 1.34\left(\mathrm{~d},{ }^{3} J_{H-P}=12.33 \mathrm{~Hz}, 18 \mathrm{H}\right)$, $0.50\left(\operatorname{brq}(1: 1 ; 1: 1),{ }^{1} J_{H-B}=85 \mathrm{~Hz}, 3 \mathrm{H}\right)$.

\section{4-(Di-t-butylphosphino)- $N, N$-dimethylpiperidinium iodide-borane adduct ([t-Bu-Pip-}

phos•BH ${ }_{3}$ ]I. 4-(Di-t-butylphospino)- $N$-methylpiperidine-borane adduct (1.81 g, 7.04 mmol) was dissolved in $100 \mathrm{~mL}$ THF and methyl iodide $(1.10 \mathrm{~mL}, 17.7 \mathrm{mmol})$ was added. The reaction was refluxed overnight during which time a white precipitate formed. The precipitate was filtered off and washed with ether. The crude product was recrystallized from boiling methanol to give large, colorless, blocks (1.39 g, $50 \%) .{ }^{1} \mathrm{H}$ NMR ( $\left.\mathrm{CD}_{3} \mathrm{OD}, 360 \mathrm{MHz}\right): \delta 3.58-3.50(\mathrm{~m}, 4 \mathrm{H}), 3.33$ $(\mathrm{s}, 3 \mathrm{H}), 3.13(\mathrm{~s}, 3 \mathrm{H}), 2.51-2.42(\mathrm{~m}, 2 \mathrm{H}), 2.35-2.20(\mathrm{~m}, 3 \mathrm{H}), 1.39\left(\mathrm{~d},{ }^{3} J_{H-P}=20.35 \mathrm{~Hz}, 18 \mathrm{H}\right), 0.40$ (brq $\left.(1: 1: 1: 1),{ }^{1} J_{H-B}=77.2 \mathrm{~Hz}, 3 \mathrm{H}\right) .{ }^{13} \mathrm{C} \mathrm{NMR}\left(\mathrm{CD}_{3} \mathrm{OD}, 90.6 \mathrm{MHz}\right): \delta 64.4\left(\mathrm{~d}, J_{C-P}=9.25 \mathrm{~Hz}\right)$, $57.15\left(\mathrm{~d}, J_{C-P}=3.98 \mathrm{~Hz}\right), 57.10\left(\mathrm{~d}, J_{C-P}=3.98 \mathrm{~Hz}\right), 35.2\left(\mathrm{~d}, J_{C-P}=25.19 \mathrm{~Hz}\right), 30.5\left(\mathrm{~d}, J_{C-P}=23.88 \mathrm{~Hz}\right)$, 
29.5, 25.5. ${ }^{31} \mathrm{P}$ NMR $\left(\mathrm{CD}_{3} \mathrm{OD}, 202.5 \mathrm{MHz}\right): \delta 49.3$ (s). Calculated for $\mathrm{C}_{15} \mathrm{H}_{36} \mathrm{BINP}: \mathrm{C}, 45.14$;

H, 9.09; N, 3.51. Found: C, 44.93; H, 8.95; N, 3.46 .

\section{4-(Di-t-butylphosphino)- $N, N$-dimethylpiperidinium chloride-borane adduct ([t-Bu-Pip-}

phos $\left.\mathbf{B H}_{3}\right] \mathbf{C l}$. The recrystallized iodide was dissolved in warm methanol $(50 \mathrm{~mL})$ and then 50 $\mathrm{mL}$ of water was added followed by $35 \mathrm{~g}$ of Amberlite ${ }^{\circledR} \mathrm{IR} 400(\mathrm{Cl})$ anion exchange resin. The mixture was stirred for 12 hours and then the resin was filtered off. Fresh resin was added to the filtrate. After an additional 12 hours, the resin was filtered off and fresh resin was added again along with $2 \mathrm{~mL}$ of $1 \mathrm{M} \mathrm{HCl}$. After an additional 12 hours, the resin was filtered off and the solvent was removed under reduced pressure to give a waxy solid. The solid was taken up in a small amount of methanol and precipitated by addition of ether. The solid was filtered off and washed with ether then dried in vacuo. Recovered $975.2 \mathrm{mg}(91 \%)$ of $\left[t-\mathrm{Bu}-\mathrm{Pip}-\mathrm{phos} \bullet \mathrm{BH}_{3}\right] \mathrm{Cl}$ as a fluffy, white solid. ${ }^{1} \mathrm{H}$ NMR $\left(\mathrm{D}_{2} \mathrm{O}, 360 \mathrm{MHz}\right)$ : $\delta 3.62(\mathrm{brd}, J=12.33 \mathrm{~Hz}, 2 \mathrm{H}), 3.44$ (brt, $J=11.72,13.56 \mathrm{~Hz}, 2 \mathrm{H}), 3.22(\mathrm{~s}, 3 \mathrm{H}), 3.15(\mathrm{~s}, 3 \mathrm{H}), 2.60-2.51(\mathrm{~m}, 2 \mathrm{H}), 2.44-2.25(\mathrm{~m}, 3 \mathrm{H}), 1.40$ $\left(\mathrm{d},{ }^{3} J_{H-P}=12.95 \mathrm{~Hz}, 18 \mathrm{H}\right) 0.40\left(\mathrm{brq},{ }^{1} J_{H-B}=84.5 \mathrm{~Hz}, 3 \mathrm{H}\right) .{ }^{13} \mathrm{C}$ NMR $\left(\mathrm{D}_{2} \mathrm{O}, 90.6 \mathrm{MHz}\right): \delta 63.8(\mathrm{~d}$, $\left.J_{C-P}=10.62 \mathrm{~Hz}\right), 57.0,47.8,34.0\left(\mathrm{~d}, J_{C-P}=16.54 \mathrm{~Hz}\right), 29.3\left(\mathrm{~d}, J_{C-P}=26.54 \mathrm{~Hz}\right), 28.7,24.3$.

4-(Di-t-butylphosphino)- $N, N$-dimethylpiperidinium chloride ( $t$-Bu-Pip-phos). [t-Bu-Pipphos $\left.\bullet \mathrm{BH}_{3}\right] \mathrm{Cl}$ (953 mg, $3.10 \mathrm{mmol}$ ) was taken up in dry, degassed morpholine under nitrogen. The suspension was heated to $100{ }^{\circ} \mathrm{C}$ and stirred for 4 hours. The reaction was then allowed to cool to room temperature to give a suspension of a white crystalline material. THF (50 mL) was added to complete the precipitation of the product. The solvent was removed via cannula and the solid was washed with $20 \mathrm{~mL}$ of THF and dried in vacuo. The crude material was transferred to a sublimation apparatus in a drybox and heated under vacuum $\left(0.05\right.$ torr) at $110{ }^{\circ} \mathrm{C}$ for 3 hours. A small amount of morpholine-borane adduct sublimed during this time. The pure product was 
recovered as fine, colorless needles (532.3 mg, $58 \%)$. ${ }^{1} \mathrm{H}$ NMR $\left(\mathrm{D}_{2} \mathrm{O}, 360 \mathrm{MHz}\right): \delta 3.56$ (brd, $J=11.1 \mathrm{~Hz}, 2 \mathrm{H}), 3.42$ (brt, $J=12.95 \mathrm{~Hz}, 2 \mathrm{H}), 3.20$ (s, 3H), 3.13 (s, 3H), 2.42-2.20 (m, 4H), 2.10$1.98(\mathrm{~m}, 1 \mathrm{H}), 1.28\left(\mathrm{~d},{ }^{3} J_{H-P}=9.87 \mathrm{~Hz}, 18 \mathrm{H}\right) .{ }^{13} \mathrm{C} \mathrm{NMR}\left(\mathrm{D}_{2} \mathrm{O}, 90.6 \mathrm{MHz}\right): \delta 64.3\left(\mathrm{~d}, J_{C-P}=10.61\right.$ $\mathrm{Hz}), 57.0,47.6,32.8\left(\mathrm{~d}, J_{C-P}=15.92 \mathrm{~Hz}\right), 30.4\left(\mathrm{~d}, J_{C-P}=11.94 \mathrm{~Hz}\right), 29.7\left(\mathrm{~d}, J_{C-P}=21.23 \mathrm{~Hz}\right), 26.7(\mathrm{~d}$, $\left.J_{C-P}=11.94 \mathrm{~Hz}\right) .{ }^{31} \mathrm{P}$ NMR $\left(\mathrm{D}_{2} \mathrm{O}, 202.5 \mathrm{MHz}\right): \delta 40.0$ (s). Calculated for $\mathrm{C}_{15} \mathrm{H}_{33} \mathrm{ClNP}: \mathrm{C}, 61.31$; H, 11.32; N, 4.77. Found: C, 60.38; H, 11.12; N, 4.76. Carbon analysis was consistently low. 4-(Dicyclohexylphosphino)- $N, N$-dimethylpiperidinium chloride (Cy-Pip-phos). Cy-Pipphos was prepared as described by Grubbs. ${ }^{2}{ }^{1} \mathrm{H}$ NMR $\left(\mathrm{D}_{2} \mathrm{O}, 360 \mathrm{MHz}\right): \delta 3.55$ (brd, $12.33 \mathrm{~Hz}$, 2H), 3.38 (brt, $J=11.09,12.57 \mathrm{~Hz}, 2 \mathrm{H}), 3.19$ (s, 3H), 3.11 (s, 3H), 2.15-1.70 (m, $17 \mathrm{H}), 1.38-1.20$ (m, 10H). ${ }^{13} \mathrm{C}$ NMR (D $\left.2 \mathrm{O}, 90.6 \mathrm{MHz}\right): \delta 63.4\left(\mathrm{~d}, J_{\mathrm{C}-\mathrm{P}}=10.17 \mathrm{~Hz}\right), 56.9,47.6,31,4\left(\mathrm{~d}, J_{\mathrm{C}-\mathrm{P}}=8.48\right.$ $\mathrm{Hz}), 31.1\left(\mathrm{~d}, J_{\mathrm{C}-\mathrm{P}}=3.39 \mathrm{~Hz}\right), 30.0\left(\mathrm{~d}, J_{\mathrm{C}-\mathrm{P}}=5.93 \mathrm{~Hz}\right), 27.6\left(\mathrm{~d}, J_{\mathrm{C}-\mathrm{P}}=10.18 \mathrm{~Hz}\right), 26.6,25.9\left(\mathrm{~d}, J_{\mathrm{C}-}\right.$ $\left.{ }_{\mathrm{P}}=12.72 \mathrm{~Hz}\right), 25.0\left(\mathrm{~d}, J_{\mathrm{C}-\mathrm{P}}=12.71 \mathrm{~Hz}\right) .{ }^{31} \mathrm{P}$ NMR $\left(\mathrm{D}_{2} \mathrm{O}, 202.5 \mathrm{MHz}\right): \delta 7.6(\mathrm{~s})$.

General procedure for measurement of $\chi$ electronic parameter. Solutions of $\mathrm{LNi}(\mathrm{CO})_{3}$ complexes were prepared according to the procedure reported by Grubbs. ${ }^{2}$ Thus, the phosphine (0.18 mmol) was dissolved in $3 \mathrm{~mL}$ of $\mathrm{CH}_{2} \mathrm{Cl}_{2}$ in a drybox under nitrogen. $\mathrm{Ni}(\mathrm{CO})_{4}(20 \mu \mathrm{L}, 0.18$ mmol, CAUTION, highly toxic, volatile liquid) was added to the solution and the mixture was sealed and allowed to stir for 15 minutes. At this time, $100 \mu \mathrm{L}$ of the reaction mixture was diluted to $1 \mathrm{~mL}$ with $\mathrm{CH}_{2} \mathrm{Cl}_{2}$. The IR spectra were obtained in an air-free solution cell $(\mathrm{NaCl}$ windows). ( $t$-Bu-Pip-phos) Ni(CO) $)_{3}: 2060.3 \mathrm{~cm}^{-1}$. (t-Bu-Amphos) $\mathrm{Ni}(\mathrm{CO})_{3}: 2063.6 \mathrm{~cm}^{-1}$. (CyPip-phos)Ni(CO) $)_{3}: 2061.9 \mathrm{~cm}^{-1}$.

General procedure for generation of $\mathrm{L}_{2} \mathbf{P d}(0)$ in the presence of phenylboronic acid. In a drybox, $\mathrm{Pd}(\mathrm{OAc})_{2}(0.035 \mathrm{mmol})$, ligand $(0.5-3$ equiv relative to $\mathrm{Pd}), \mathrm{Na}_{2} \mathrm{CO}_{3}(0.67 \mathrm{mmol})$, and phenylboronic acid $(0.29 \mathrm{mmol})$ were added to a screw-cap vial sealed with a septum. A 
degassed solution of acetonitrile:water 1:1 (1 mL) was added via syringe under nitrogen purge. The reaction was allowed to stir at room temperature for 5 minutes. After 5 minutes formation of a biphasic solution was observed, and each layer was taken up by syringe and added to a septum sealed NMR tube under nitrogen and analyzed by ${ }^{31} \mathrm{P}$ NMR spectroscopy. Samples prepared from 2:1 ratios of $t$-Bu-Pip-phos or $t$-Bu-Amphos to $\mathrm{Pd}(\mathrm{OAc})_{2}$ were also characterized by ${ }^{1} \mathrm{H}$, and ${ }^{13} \mathrm{C}$ NMR spectroscopy.

$(\boldsymbol{t} \text {-Bu-Pip-phos })_{2} \mathbf{P d}(\mathbf{0})$. An NMR sample was prepared as described above for a 2:1 ratio of $t$ Bu-Pip-phos: $\operatorname{Pd}(\mathrm{OAc})_{2}$. The aqueous-phase showed no resonances in the ${ }^{31} \mathrm{P}$ NMR spectrum and was not further analyzed. Acetonitrile layer: ${ }^{1} \mathrm{H} \mathrm{NMR}\left(\mathrm{CD}_{3} \mathrm{CN} / \mathrm{D}_{2} \mathrm{O}, 360 \mathrm{MHz}\right): \delta 3.28$ (brd, $J=11.72 \mathrm{~Hz}, 2 \mathrm{H}), 3.09$ (brt, $J=12.96 \mathrm{~Hz}, 2 \mathrm{H}), 2.95$ (s, 3H), 2.84 (s, 3H), 2.50 (brd, $J=12.33 \mathrm{~Hz}$, 2.09 (brq, $J=13.55 \mathrm{~Hz}, 2 \mathrm{H}), 1.82\left(\mathrm{brm}, 1 \mathrm{H}\right.$ ), 1.32 (virtual triplet (vt, $J_{H-P}=6.16 \mathrm{~Hz}, 18 \mathrm{H}$ ). ${ }^{13} \mathrm{C}$ $\operatorname{NMR}\left(\mathrm{CD}_{3} \mathrm{CN} / \mathrm{D}_{2} \mathrm{O}, 90.6 \mathrm{MHz}\right): \delta 64.1\left(\mathrm{vt}, J_{C-P}=5.31 \mathrm{~Hz}\right), 56.9,47.8,36.2\left(\mathrm{vt}, J_{C-P}=2.65 \mathrm{~Hz}\right)$, 33.0, $31.7\left(\mathrm{vt}, J_{C-P}=6.63,5.31 \mathrm{~Hz}\right), 28.3\left(\mathrm{vt}, J_{C-P}=5.31 \mathrm{~Hz}\right) .{ }^{31} \mathrm{P} \mathrm{NMR}\left(\mathrm{CD}_{3} \mathrm{CN} / \mathrm{D}_{2} \mathrm{O}, 202.5 \mathrm{MHz}\right)$ : $\delta 70.4(s)$.

( $t$-Bu-Amphos $)_{2} \mathbf{P d}(\mathbf{0})$. An NMR sample was prepared as described above for a 2:1 ratio of $t$ Bu-Amphos:Pd(OAc) $)_{2}$. The aqueous-phase showed no resonances by ${ }^{31} \mathrm{P}$ NMR and was not further analyzed. Acetonitrile layer: ${ }^{1} \mathrm{H} N M R\left(\mathrm{CD}_{3} \mathrm{CN} / \mathrm{D}_{2} \mathrm{O}, 360 \mathrm{MHz}\right): \delta 3.64(\mathrm{brm}, 2 \mathrm{H}), 2.98$ (s, 9H), 1.75 (brm, 2H), 1.26 (vt, $\left.J_{H-P}=6.38 \mathrm{~Hz}, 18 \mathrm{H}\right) .{ }^{13} \mathrm{C} \mathrm{NMR}\left(\mathrm{CD}_{3} \mathrm{CN} / \mathrm{D}_{2} \mathrm{O}, 90.6 \mathrm{MHz}\right): \delta$ $69.9(\mathrm{vt}, J=17.65,18.03 \mathrm{~Hz}), 53.5\left(\mathrm{t}(1: 1: 1), J_{\mathrm{C}-\mathrm{N}}=4.16 \mathrm{~Hz}\right), 35.5$ (vt, $\left.J=5.55 \mathrm{~Hz}\right), 30.6$ (vt, $J=5.55$ $\mathrm{Hz})$, 16.7. ${ }^{31} \mathrm{P} \mathrm{NMR}\left(\mathrm{CD}_{3} \mathrm{CN} / \mathrm{D}_{2} \mathrm{O}, 202,5 \mathrm{MHz}\right): \delta 55.8(\mathrm{~s})$.

(Cy-Pip-phos) $)_{2} \mathbf{P d}(\mathbf{0})$. An NMR sample was prepared as described above for a 1:1 ratio CyPip-phos:Pd(OAc) $)_{2} \quad$ Acetonitrile: ${ }^{31} \mathrm{P}$ NMR $\left(\mathrm{CD}_{3} \mathrm{CN} / \mathrm{D}_{2} \mathrm{O}, 202.5 \mathrm{MHz}\right): \quad \delta 55.0(\mathrm{~s}$, 
integral=0.07), $47.4(\mathrm{~s}$, integral=0.11), $42.6(\mathrm{~s}$, integral=0.17), $39.6 \mathrm{ppm}(\mathrm{s}$, integral=1.0), $26.3(\mathrm{~s}$, integral=0.06), $21.2(\mathrm{~s}$, integral=0.02).

General procedure for preparation of $L_{2} P d(0)$ in the presence of sodium formate. In the drybox, a vial was charged with $\mathrm{Pd}(\mathrm{OAc})_{2}(0.035 \mathrm{mmol})$, ligand $(0.5-3$ equiv. to $\mathrm{Pd})$, and sodium formate $(0.7 \mathrm{mmol})$. A degassed solution of acetonitrile:water 1:1 (1 mL) was added by syringe to the sealed vial containing the solid reagents. The reaction was allowed to stir at room temperature for 5 minutes at which point the solution was transferred to a septum sealed NMR tube by syringe and analyzed by ${ }^{31} \mathrm{P}$ NMR. The sample produced from a 1:1 ratio of Cy-pipphos: $\mathrm{Pd}(\mathrm{OAc})_{2}$ was also analyzed by ${ }^{1} \mathrm{H}$ and ${ }^{13} \mathrm{C}$ NMR.

$(\boldsymbol{t} \text {-Bu-Pip-phos })_{2} \mathbf{P d}(\mathbf{0})$. An NMR sample was prepared as described above with a 1:1 ratio of $t$-Bu-Pip-phos:Pd(OAc) $)_{2} .{ }^{31} \mathrm{P} \mathrm{NMR}\left(\mathrm{CH}_{3} \mathrm{CN} / \mathrm{H}_{2} \mathrm{O}, 202.5 \mathrm{MHz}\right): \delta 70$ (s).

(Cy-Pip-phos) $)_{2} \mathbf{P d}(\mathbf{0})$. A 1:1 mixture of Cy-Pip-phos:Pd(OAc) $)_{2}$ was reduced by sodium formate as described above. ${ }^{13} \mathrm{C}$ NMR $\left(\mathrm{CD}_{3} \mathrm{CN} / \mathrm{D}_{2} \mathrm{O}, 90.6 \mathrm{MHz}\right): \delta 63.4,57.1,47.8,33.3\left(\mathrm{vt}, J_{\mathrm{C}-}\right.$ $\left.{ }_{\mathrm{P}}=10.61 \mathrm{~Hz}\right), 30.5 .30 .2,27.9\left(\mathrm{~d}, J_{C-P}=14.6 \mathrm{~Hz}\right), 26.7,24.3,23.6$. A second minor set of resonances with similar pattern was also observed. Free acetate was also observed at 181.5 and 23.6 ppm. ${ }^{13} \mathrm{P}$ NMR $\left(\mathrm{CD}_{3} \mathrm{CN} / \mathrm{D}_{2} \mathrm{O}, 202.5 \mathrm{MHz}\right): \delta 44$ (s), 41 (s, minor).

Complexation of $\boldsymbol{t}$-Bu-Amphos with $\mathbf{P d}(\mathrm{OAc})_{2}$. In the drybox, $\mathrm{Pd}(\mathrm{OAc})_{2}(9.0 \mathrm{mg}, 0.04$ mmol) and $t$-Bu-Amphos $(20.7 \mathrm{mg}, 0.08 \mathrm{mmol})$ were added to a screw-cap vial, which was sealed with a septum and dissolved in degassed $\mathrm{D}_{2} \mathrm{O}$. The reaction was allowed to stir at room temperature for 5 minutes, during which time a pale yellow precipitate formed. The $\mathrm{D}_{2} \mathrm{O}$ solution showed resonances for $t$-Bu-Pip-phos and two new species at 37 and $38 \mathrm{ppm}$ in the ${ }^{31} \mathrm{P}$ NMR spectrum. The solid precipitate was sparingly soluble in acetonitrile. $A{ }^{31} \mathrm{P}$ NMR spectrum of this mixture showed free $t$-Bu-Amphos and a pair of resonances at 38 and $40 \mathrm{ppm}$. 
trans-(Cy-Pip-phos) ${ }_{2} \mathbf{P d C l}_{2}$. In the drybox, $\mathrm{Na}_{2} \mathrm{PdCl}_{4}(3.0 \mathrm{mg}, 0.020 \mathrm{mmol})$ and Cy-Pip-phos (13.8 $\mathrm{mg}, 0.040 \mathrm{mmol})$ were added to a screw-cap vial, which was sealed with a septum. A degassed solution of acetonitrile:water 1:1 (1 mL) was added by syringe under nitrogen. The reaction was allowed to stir at room temperature for 5 minutes, and then analyzed by ${ }^{31} \mathrm{P}$, ${ }^{1} \mathrm{H}$, and ${ }^{13} \mathrm{C}$ NMR spectroscopy. ${ }^{1} \mathrm{H}$ NMR $\left(\mathrm{CD}_{3} \mathrm{CN} / \mathrm{D}_{2} \mathrm{O}, 360 \mathrm{MHz}\right): \delta 3,42(\mathrm{~d}, J=12.76 \mathrm{~Hz}, 2 \mathrm{H}), 3.19$ (brt, J=6.76, $6.11 \mathrm{~Hz}, 2 \mathrm{H}), 3.05(\mathrm{~s}, 3 \mathrm{H}), 3.02(\mathrm{~s}, 3 \mathrm{H}), 2.39-2.58(\mathrm{~m}, 4 \mathrm{H}), 1,90-2.05(\mathrm{~m}$, overlapped with solvent peak), 1.58-1.76 (m, $10 \mathrm{H}), 1.18-1.29(\mathrm{~m}, 6 \mathrm{H}){ }^{13} \mathrm{C} \mathrm{NMR}\left(\mathrm{CD}_{3} \mathrm{CN}_{2} \mathrm{D}_{2} \mathrm{O}\right.$, 90.6 MHz): $\delta 62.6,56.3,47.132 .5\left(\mathrm{vt}, J_{C-P}=9.71 \mathrm{~Hz}\right), 29.7,29.4,27.1\left(\mathrm{~d}, J_{C-P}=9.71 \mathrm{~Hz}\right), 26.0$, 22.9. ${ }^{31} \mathrm{P} \mathrm{NMR}\left(\mathrm{CD}_{3} \mathrm{CN} / \mathrm{D}_{2} \mathrm{O}, 202.5 \mathrm{MHz}\right): \delta 28.3(\mathrm{~s})$.

\section{References}

(1) Imamoto, T.; Oshiki, T.; Onozawa, T.; Kusumoto, T.; Sato, K. J. Am. Chem. Soc. 1990, $112,5244-5252$.

(2) Mohr, B.; Lynn, D. M.; Grubbs, R. H. Organometallics 1996, 15, 4317-4325.

(3) Sheldrick, G. M., SHELXTL, Version 6.14, Brüker Advanced X-Ray Solutions, Inc., 2000.

(4) Sheldrick, G. M., Program for Semiempirical Absorption Correlation of Area Detector Data, University of Göttingen, Germany, 1996. 


\section{X-Ray Crystallographic Data}

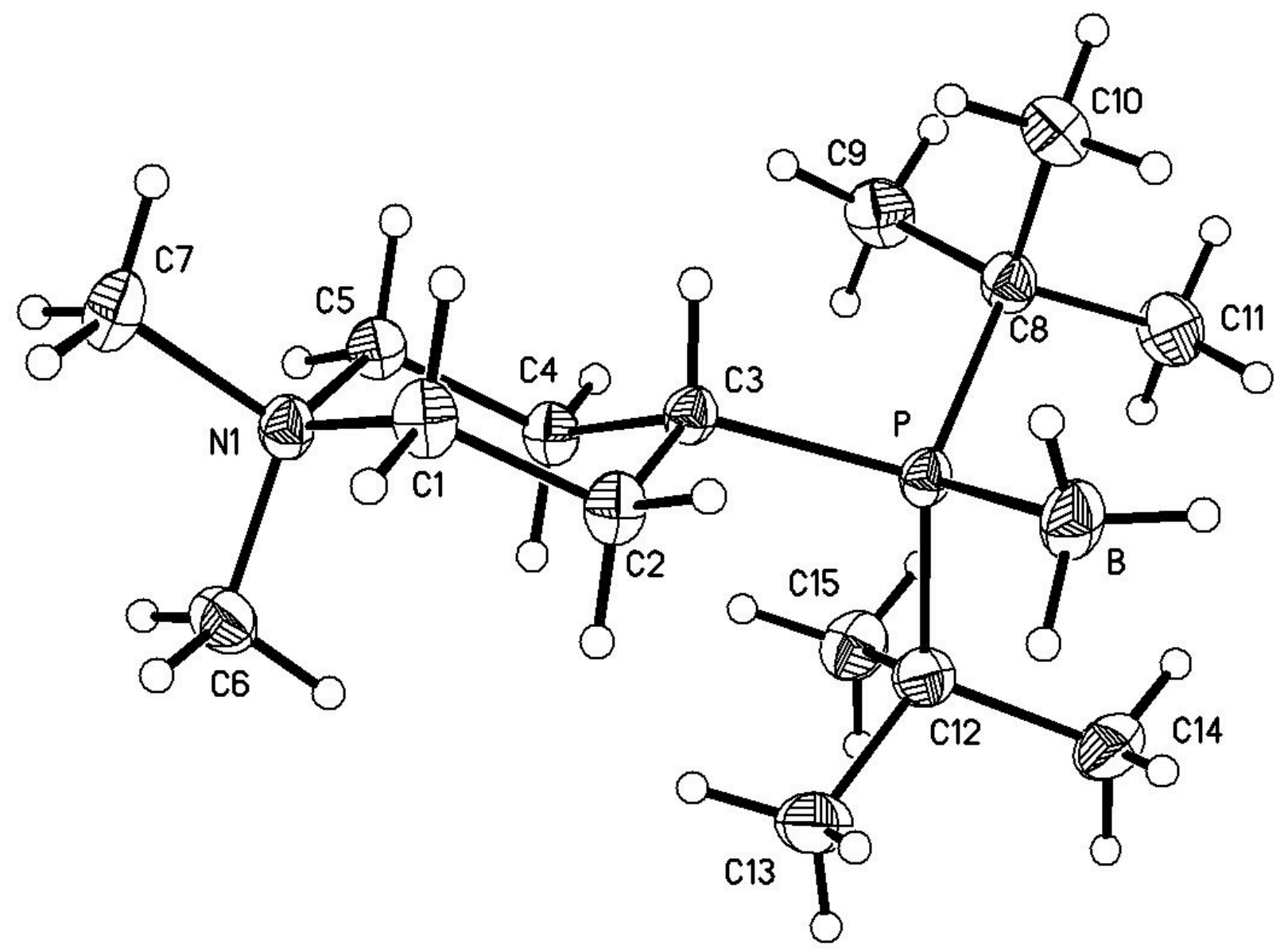

ORTEP digram of $\left[t\right.$-Bu-Pip-phos $\left.\bullet \mathrm{BH}_{3}\right] \mathrm{I}$ drawn with thermal ellipsoids at the $50 \%$ level. 
Table 1. Crystal data and structure refinement for $\left[t-\mathrm{Bu}-\mathrm{Pip}-\mathrm{phos} \bullet \mathrm{BH}_{3}\right] \mathrm{I}$

Empirical formula

Formula weight

Temperature

Wavelength

Crystal system

Space group

Unit cell dimensions

Volume

$\mathrm{Z}$

Density (calculated)

Absorption coefficient

$\mathrm{F}(000)$

Crystal size

Theta range for data collection

Index ranges

Reflections collected

Independent reflections

Completeness to theta $=$

Absorption correction

Refinement method

Data / restraints / parameters

Goodness-of-fit on $\mathrm{F}^{2}$

Final R indices

$\mathrm{R}$ indices (all data)

Extinction coefficient

Largest diff. peak and hole
$\mathrm{C}_{15} \mathrm{H}_{36} \mathrm{BINP}$

399.13

173(2) K

$0.71073 \AA$

Monoclinic

$\mathrm{P} 2(1) / \mathrm{c}$

$\mathrm{a}=11.7778(17) \AA \quad \alpha=90^{\circ}$

$\mathrm{b}=11.5772(17) \AA \quad \beta=90.233(3)^{\circ}$

$\mathrm{c}=14.397(2) \AA \quad \gamma=90^{\circ}$

1963.1(5) $\AA^{3}$

4

$1.350 \mathrm{Mg} / \mathrm{m}^{3}$

$1.704 \mathrm{~mm}^{-1}$

824

$0.24 \times 0.28 \times 0.48 \mathrm{~mm}^{3}$

2.26 to $23.30^{\circ}$

$-11<=\mathrm{h}<=13,-12<=\mathrm{k}<=12,-16<=\mathrm{l}<=13$

8409

$2824[\mathrm{R}(\mathrm{int})=0.0253]$

$23.30^{\circ} 99.6 \%$

\section{SADABS}

Full-matrix least-squares on $\mathrm{F}^{2}$

2824 / 0 / 185

1.118

[I $>2 \operatorname{sigma}(\mathrm{I})] \quad \mathrm{R} 1=0.0204, \mathrm{wR} 2=0.0465$

$\mathrm{R} 1=0.0212, \mathrm{wR} 2=0.0470$

$0.0051(2)$

0.385 and -0.314 e. $\AA^{-3}$ 


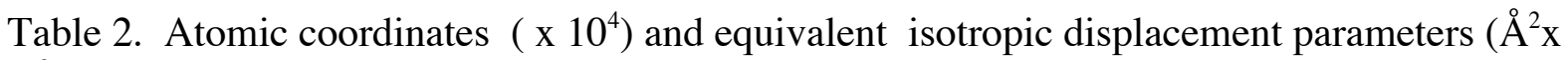
$\left.10^{3}\right)$ for $\left[t-\mathrm{Bu}\right.$-Pip-phos $\left.\bullet \mathrm{BH}_{3}\right]$ I. $\mathrm{U}(\mathrm{eq})$ is defined as one third of the trace of the orthogonalized Uij tensor.

\begin{tabular}{lllll}
\hline \multicolumn{1}{c}{$\mathrm{x}$} & $\mathrm{y}$ & $\mathrm{z}$ & $\mathrm{U}(\mathrm{eq})$ \\
\hline $\mathrm{I}(1)$ & $1304(1)$ & $2334(1)$ & $8851(1)$ & $29(1)$ \\
$\mathrm{P}(1)$ & $3120(1)$ & $-1775(1)$ & $6698(1)$ & $18(1)$ \\
$\mathrm{N}(1)$ & $1400(1)$ & $-5503(2)$ & $6432(1)$ & $20(1)$ \\
$\mathrm{C}(1)$ & $1326(2)$ & $-4788(2)$ & $7307(1)$ & $22(1)$ \\
$\mathrm{C}(2)$ & $2248(2)$ & $-3887(2)$ & $7381(1)$ & $22(1)$ \\
$\mathrm{C}(3)$ & $2226(2)$ & $-3081(2)$ & $6535(1)$ & $18(1)$ \\
$\mathrm{C}(4)$ & $2370(2)$ & $-3816(2)$ & $5656(1)$ & $20(1)$ \\
$\mathrm{C}(5)$ & $1431(2)$ & $-4709(2)$ & $5598(1)$ & $20(1)$ \\
$\mathrm{C}(6)$ & $2411(2)$ & $-6288(2)$ & $6443(2)$ & $27(1)$ \\
$\mathrm{C}(7)$ & $355(2)$ & $-6237(2)$ & $6372(2)$ & $27(1)$ \\
$\mathrm{C}(8)$ & $2399(2)$ & $-633(2)$ & $5980(1)$ & $21(1)$ \\
$\mathrm{C}(9)$ & $2007(2)$ & $-1050(2)$ & $5016(2)$ & $29(1)$ \\
$\mathrm{C}(10)$ & $1345(2)$ & $-240(2)$ & $6521(2)$ & $30(1)$ \\
$\mathrm{C}(11)$ & $3177(2)$ & $413(2)$ & $5862(2)$ & $32(1)$ \\
$\mathrm{C}(12)$ & $4613(2)$ & $-2074(2)$ & $6317(2)$ & $25(1)$ \\
$\mathrm{C}(13)$ & $5014(2)$ & $-3194(2)$ & $6795(2)$ & $33(1)$ \\
$\mathrm{C}(14)$ & $5387(2)$ & $-1097(2)$ & $6677(2)$ & $34(1)$ \\
$\mathrm{C}(15)$ & $4768(2)$ & $-2201(2)$ & $5268(2)$ & $30(1)$ \\
$\mathrm{B}(1)$ & $3105(2)$ & $-1353(2)$ & $7998(2)$ & $27(1)$ \\
\hline
\end{tabular}


Table 3. Bond lengths $[\AA]$ and angles $\left[{ }^{\circ}\right]$ for $\left[t\right.$-Bu-Pip-phos $\left.\bullet \mathrm{BH}_{3}\right] \mathrm{I}$.

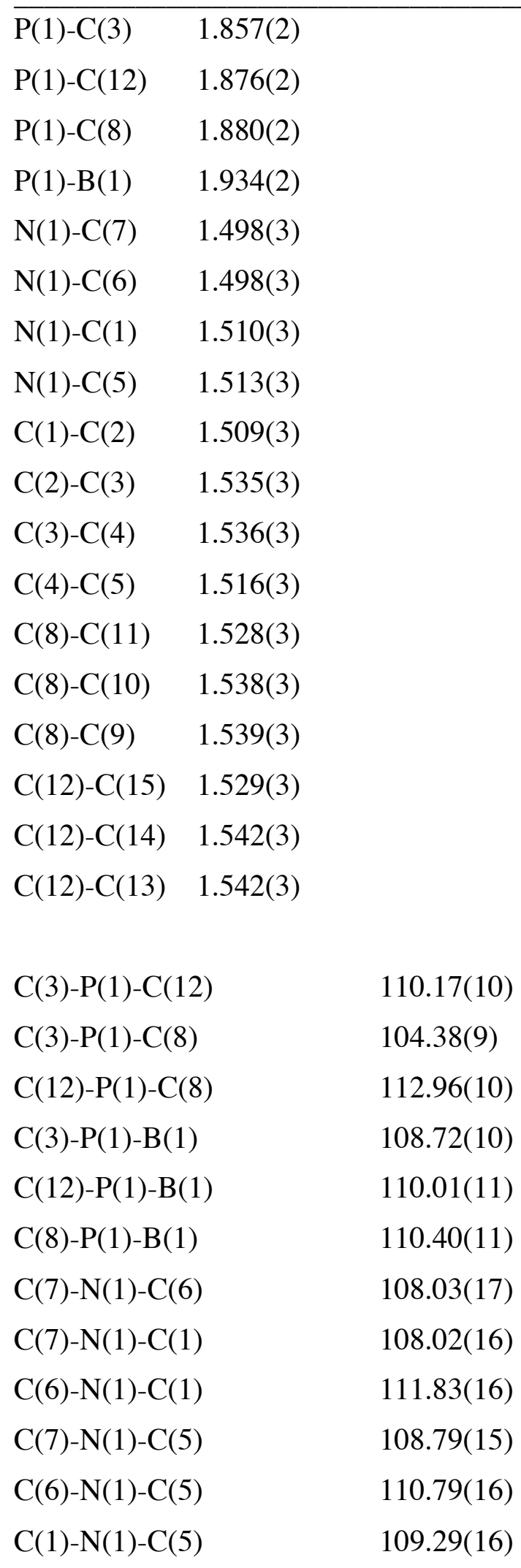




$\begin{array}{ll}\mathrm{C}(2)-\mathrm{C}(1)-\mathrm{N}(1) & 113.23(17) \\ \mathrm{C}(1)-\mathrm{C}(2)-\mathrm{C}(3) & 110.72(17) \\ \mathrm{C}(2)-\mathrm{C}(3)-\mathrm{C}(4) & 108.41(17) \\ \mathrm{C}(2)-\mathrm{C}(3)-\mathrm{P}(1) & 112.75(14) \\ \mathrm{C}(4)-\mathrm{C}(3)-\mathrm{P}(1) & 119.40(14) \\ \mathrm{C}(5)-\mathrm{C}(4)-\mathrm{C}(3) & 109.89(17) \\ \mathrm{N}(1)-\mathrm{C}(5)-\mathrm{C}(4) & 113.01(16) \\ \mathrm{C}(11)-\mathrm{C}(8)-\mathrm{C}(10) & 107.90(19) \\ \mathrm{C}(11)-\mathrm{C}(8)-\mathrm{C}(9) & 109.06(18) \\ \mathrm{C}(10)-\mathrm{C}(8)-\mathrm{C}(9) & 108.02(18) \\ \mathrm{C}(11)-\mathrm{C}(8)-\mathrm{P}(1) & 110.45(15) \\ \mathrm{C}(10)-\mathrm{C}(8)-\mathrm{P}(1) & 107.07(14) \\ \mathrm{C}(9)-\mathrm{C}(8)-\mathrm{P}(1) & 114.13(15) \\ \mathrm{C}(15)-\mathrm{C}(12)-\mathrm{C}(14) & 109.30(19) \\ \mathrm{C}(15)-\mathrm{C}(12)-\mathrm{C}(13) & 108.8(2) \\ \mathrm{C}(14)-\mathrm{C}(12)-\mathrm{C}(13) & 106.66(19) \\ \mathrm{C}(15)-\mathrm{C}(12)-\mathrm{P}(1) & 115.01(16) \\ \mathrm{C}(14)-\mathrm{C}(12)-\mathrm{P}(1) & 108.67(16) \\ \mathrm{C}(13)-\mathrm{C}(12)-\mathrm{P}(1) & 108.06(15)\end{array}$

Symmetry transformations used to generate equivalent atoms: 
Table 4. Anisotropic displacement parameters $\left(\AA^{2} \times 10^{3}\right)$ for $\left[t\right.$-Bu-Pip-phos $\left.\bullet \mathrm{BH}_{3}\right] \mathrm{I}$. The anisotropic displacement factor exponent takes the form: $-2 \pi 2\left[\mathrm{~h} 2 \mathrm{a} * 2 \mathrm{U} 11+\ldots+2 \mathrm{~h} \mathrm{k} \mathrm{a} \mathrm{b}^{*}\right.$ $\mathrm{U} 12$ ]

\begin{tabular}{lllllll}
\hline & $\mathrm{U} 11$ & $\mathrm{U} 22$ & $\mathrm{U} 33$ & $\mathrm{U} 23$ & $\mathrm{U} 13$ & $\mathrm{U} 12$ \\
\hline $\mathrm{I}(1)$ & $30(1)$ & $29(1)$ & $28(1)$ & $11(1)$ & $-2(1)$ & $-3(1)$ \\
$\mathrm{P}(1)$ & $21(1)$ & $17(1)$ & $15(1)$ & $0(1)$ & $-2(1)$ & $-3(1)$ \\
$\mathrm{N}(1)$ & $24(1)$ & $17(1)$ & $19(1)$ & $2(1)$ & $-2(1)$ & $-3(1)$ \\
$\mathrm{C}(1)$ & $32(1)$ & $22(1)$ & $13(1)$ & $1(1)$ & $2(1)$ & $-3(1)$ \\
$\mathrm{C}(2)$ & $29(1)$ & $21(1)$ & $15(1)$ & $1(1)$ & $-1(1)$ & $-4(1)$ \\
$\mathrm{C}(3)$ & $20(1)$ & $18(1)$ & $16(1)$ & $0(1)$ & $-2(1)$ & $-2(1)$ \\
$\mathrm{C}(4)$ & $26(1)$ & $19(1)$ & $16(1)$ & $2(1)$ & $0(1)$ & $-2(1)$ \\
$\mathrm{C}(5)$ & $26(1)$ & $21(1)$ & $14(1)$ & $1(1)$ & $-3(1)$ & $-1(1)$ \\
$\mathrm{C}(6)$ & $31(1)$ & $22(1)$ & $29(1)$ & $2(1)$ & $-4(1)$ & $4(1)$ \\
$\mathrm{C}(7)$ & $30(1)$ & $27(1)$ & $26(1)$ & $1(1)$ & $-2(1)$ & $-11(1)$ \\
$\mathrm{C}(8)$ & $25(1)$ & $18(1)$ & $21(1)$ & $2(1)$ & $-2(1)$ & $-2(1)$ \\
$\mathrm{C}(9)$ & $38(1)$ & $29(1)$ & $22(1)$ & $6(1)$ & $-5(1)$ & $2(1)$ \\
$\mathrm{C}(10)$ & $32(1)$ & $24(1)$ & $32(1)$ & $0(1)$ & $1(1)$ & $4(1)$ \\
$\mathrm{C}(11)$ & $37(1)$ & $22(1)$ & $38(1)$ & $9(1)$ & $-1(1)$ & $-3(1)$ \\
$\mathrm{C}(12)$ & $23(1)$ & $25(1)$ & $26(1)$ & $1(1)$ & $-2(1)$ & $0(1)$ \\
$\mathrm{C}(13)$ & $25(1)$ & $33(1)$ & $40(1)$ & $4(1)$ & $-4(1)$ & $3(1)$ \\
$\mathrm{C}(14)$ & $25(1)$ & $36(1)$ & $40(1)$ & $-1(1)$ & $-6(1)$ & $-5(1)$ \\
$\mathrm{C}(15)$ & $26(1)$ & $34(1)$ & $30(1)$ & $1(1)$ & $6(1)$ & $-5(1)$ \\
$\mathrm{B}(1)$ & $36(2)$ & $27(1)$ & $19(1)$ & $-3(1)$ & $-6(1)$ & $-3(1)$ \\
& & & & & & \\
\hline
\end{tabular}


Table 5. Hydrogen coordinates $\left(\times 10^{4}\right)$ and isotropic displacement parameters $\left(\AA^{2} \times 10^{3}\right)$ for $[t$ Bu-Pip-phos $\bullet \mathrm{BH}_{3}$ ]I.

\begin{tabular}{lllll}
\hline & $\mathrm{x}$ & $\mathrm{y}$ & $\mathrm{z}$ & $\mathrm{U}(\mathrm{eq})$ \\
\hline $\mathrm{H}(1 \mathrm{~B})$ & 593 & -4406 & 7324 & 26 \\
$\mathrm{H}(1 \mathrm{~A})$ & 1373 & -5297 & 7841 & 26 \\
$\mathrm{H}(2 \mathrm{~B})$ & 2982 & -4264 & 7422 & 26 \\
$\mathrm{H}(2 \mathrm{~A})$ & 2141 & -3437 & 7943 & 26 \\
$\mathrm{H}(3 \mathrm{~A})$ & 1445 & -2791 & 6503 & 21 \\
$\mathrm{H}(4 \mathrm{~B})$ & 2345 & -3323 & 5111 & 24 \\
$\mathrm{H}(4 \mathrm{~A})$ & 3102 & -4200 & 5671 & 24 \\
$\mathrm{H}(5 \mathrm{~B})$ & 707 & -4315 & 5543 & 24 \\
$\mathrm{H}(5 \mathrm{~A})$ & 1535 & -5169 & 5041 & 24 \\
$\mathrm{H}(6 \mathrm{C})$ & 2429 & -6729 & 5879 & 41 \\
$\mathrm{H}(6 \mathrm{~B})$ & 2361 & -6802 & 6964 & 41 \\
$\mathrm{H}(6 \mathrm{~A})$ & 3092 & -5835 & 6493 & 41 \\
$\mathrm{H}(7 \mathrm{~A})$ & 367 & -6675 & 5805 & 41 \\
$\mathrm{H}(7 \mathrm{~B})$ & -306 & -5752 & 6379 & 41 \\
$\mathrm{H}(7 \mathrm{C})$ & 334 & -6756 & 6892 & 41 \\
$\mathrm{H}(9 \mathrm{C})$ & 1711 & -409 & 4669 & 44 \\
$\mathrm{H}(9 \mathrm{~B})$ & 1425 & -1624 & 5087 & 44 \\
$\mathrm{H}(9 \mathrm{~A})$ & 2640 & -1379 & 4691 & 44 \\
$\mathrm{H}(10 \mathrm{C})$ & 932 & 318 & 6160 & 44 \\
$\mathrm{H}(10 B)$ & 1577 & 102 & 7098 & 44 \\
$\mathrm{H}(10 \mathrm{~A})$ & 867 & -894 & 6641 & 44 \\
$\mathrm{H}(11 \mathrm{C})$ & 2764 & 1026 & 5563 & 48 \\
$\mathrm{H}(11 \mathrm{~B})$ & 3817 & 202 & 5488 & 48 \\
$\mathrm{H}(11 \mathrm{~A})$ & 3437 & 668 & 6460 & 48 \\
$\mathrm{H}(13 \mathrm{~A})$ & 5808 & -3308 & 6677 & 49 \\
$\mathrm{H}(13 \mathrm{~B})$ & 4591 & -3837 & 6555 & 49 \\
$\mathrm{H}(13 \mathrm{C})$ & 4894 & -3134 & 7453 & 49 \\
$\mathrm{H}(14 \mathrm{~A})$ & 6164 & -1280 & 6542 & 50 \\
& & & &
\end{tabular}




\begin{tabular}{lllll}
$\mathrm{H}(14 \mathrm{~B})$ & 5295 & -1017 & 7336 & 50 \\
$\mathrm{H}(14 \mathrm{C})$ & 5185 & -385 & 6377 & 50 \\
$\mathrm{H}(15 \mathrm{~A})$ & 5551 & -2354 & 5135 & 45 \\
$\mathrm{H}(15 \mathrm{~B})$ & 4539 & -1500 & 4965 & 45 \\
$\mathrm{H}(15 \mathrm{C})$ & 4310 & -2830 & 5046 & 45 \\
$\mathrm{H}(1 \mathrm{~B} 1)$ & 3602 & -1857 & 8339 & $47(8)$ \\
$\mathrm{H}(2 \mathrm{~B} 1)$ & 2347 & -1422 & 8234 & $41(7)$ \\
$\mathrm{H}(3 \mathrm{~B} 1)$ & 3360 & -569 & 8065 & $41(7)$ \\
\hline
\end{tabular}


Table 6. Torsion angles $\left[{ }^{\circ}\right]$ for $\left[t\right.$-Bu-Pip-phos $\left.\bullet \mathrm{BH}_{3}\right] \mathrm{I}$.

\begin{tabular}{ll}
\hline $\mathrm{C}(7)-\mathrm{N}(1)-\mathrm{C}(1)-\mathrm{C}(2)$ & $-171.88(17)$ \\
$\mathrm{C}(6)-\mathrm{N}(1)-\mathrm{C}(1)-\mathrm{C}(2)$ & $69.4(2)$ \\
$\mathrm{C}(5)-\mathrm{N}(1)-\mathrm{C}(1)-\mathrm{C}(2)$ & $-53.7(2)$ \\
$\mathrm{N}(1)-\mathrm{C}(1)-\mathrm{C}(2)-\mathrm{C}(3)$ & $57.1(2)$ \\
$\mathrm{C}(1)-\mathrm{C}(2)-\mathrm{C}(3)-\mathrm{C}(4)$ & $-57.9(2)$ \\
$\mathrm{C}(1)-\mathrm{C}(2)-\mathrm{C}(3)-\mathrm{P}(1)$ & $167.60(15)$ \\
$\mathrm{C}(12)-\mathrm{P}(1)-\mathrm{C}(3)-\mathrm{C}(2)$ & $88.31(16)$ \\
$\mathrm{C}(8)-\mathrm{P}(1)-\mathrm{C}(3)-\mathrm{C}(2)$ & $-150.15(15)$ \\
$\mathrm{B}(1)-\mathrm{P}(1)-\mathrm{C}(3)-\mathrm{C}(2)$ & $-32.32(18)$ \\
$\mathrm{C}(12)-\mathrm{P}(1)-\mathrm{C}(3)-\mathrm{C}(4)$ & $-40.71(19)$ \\
$\mathrm{C}(8)-\mathrm{P}(1)-\mathrm{C}(3)-\mathrm{C}(4)$ & $80.83(17)$ \\
$\mathrm{B}(1)-\mathrm{P}(1)-\mathrm{C}(3)-\mathrm{C}(4)$ & $-161.34(16)$ \\
$\mathrm{C}(2)-\mathrm{C}(3)-\mathrm{C}(4)-\mathrm{C}(5)$ & $58.4(2)$ \\
$\mathrm{P}(1)-\mathrm{C}(3)-\mathrm{C}(4)-\mathrm{C}(5)$ & $-170.69(14)$ \\
$\mathrm{C}(7)-\mathrm{N}(1)-\mathrm{C}(5)-\mathrm{C}(4)$ & $172.23(18)$ \\
$\mathrm{C}(6)-\mathrm{N}(1)-\mathrm{C}(5)-\mathrm{C}(4)$ & $-69.2(2)$ \\
$\mathrm{C}(1)-\mathrm{N}(1)-\mathrm{C}(5)-\mathrm{C}(4)$ & $54.5(2)$ \\
$\mathrm{C}(3)-\mathrm{C}(4)-\mathrm{C}(5)-\mathrm{N}(1)$ & $-58.4(2)$ \\
$\mathrm{C}(3)-\mathrm{P}(1)-\mathrm{C}(8)-\mathrm{C}(11)$ & $-166.96(15)$ \\
$\mathrm{C}(12)-\mathrm{P}(1)-\mathrm{C}(8)-\mathrm{C}(11)$ & $-47.28(18)$ \\
$\mathrm{B}(1)-\mathrm{P}(1)-\mathrm{C}(8)-\mathrm{C}(11)$ & $76.37(18)$ \\
$\mathrm{C}(3)-\mathrm{P}(1)-\mathrm{C}(8)-\mathrm{C}(10)$ & $75.81(16)$ \\
$\mathrm{C}(12)-\mathrm{P}(1)-\mathrm{C}(8)-\mathrm{C}(10)$ & $-164.51(14)$ \\
$\mathrm{B}(1)-\mathrm{P}(1)-\mathrm{C}(8)-\mathrm{C}(10)$ & $-40.86(18)$ \\
$\mathrm{C}(3)-\mathrm{P}(1)-\mathrm{C}(8)-\mathrm{C}(9)$ & $-43.66(18)$ \\
$\mathrm{C}(12)-\mathrm{P}(1)-\mathrm{C}(8)-\mathrm{C}(9)$ & $76.02(18)$ \\
$\mathrm{B}(1)-\mathrm{P}(1)-\mathrm{C}(8)-\mathrm{C}(9)$ & $-160.33(16)$ \\
$\mathrm{C}(3)-\mathrm{P}(1)-\mathrm{C}(12)-\mathrm{C}(15)$ & $70.81(19)$ \\
$\mathrm{C}(8)-\mathrm{P}(1)-\mathrm{C}(12)-\mathrm{C}(15)$ & $-45.5(2)$ \\
$\mathrm{B}(1)-\mathrm{P}(1)-\mathrm{C}(12)-\mathrm{C}(15)$ & $-169.34(17)$ \\
$\mathrm{C}(3)-\mathrm{P}(1)-\mathrm{C}(12)-\mathrm{C}(14)$ & $-166.32(15)$ \\
\end{tabular}




$\begin{array}{ll}\mathrm{C}(8)-\mathrm{P}(1)-\mathrm{C}(12)-\mathrm{C}(14) & 77.39(17) \\ \mathrm{B}(1)-\mathrm{P}(1)-\mathrm{C}(12)-\mathrm{C}(14) & -46.47(19) \\ \mathrm{C}(3)-\mathrm{P}(1)-\mathrm{C}(12)-\mathrm{C}(13) & -50.95(18) \\ \mathrm{C}(8)-\mathrm{P}(1)-\mathrm{C}(12)-\mathrm{C}(13) & -167.24(15) \\ \mathrm{B}(1)-\mathrm{P}(1)-\mathrm{C}(12)-\mathrm{C}(13) & 68.90(18)\end{array}$

Symmetry transformations used to generate equivalent atoms: 


\section{Computational Materials}

The local DFT optimized geometries (coordinates) of the free ligand (L), $\mathrm{LPd}(0)$ and $\mathrm{L}_{2} \mathrm{Pd}(0)$ for ligand $=$ Cy-Pip-phos, $t$-Bu-Pip-phos, $t$-Bu-Amphos, and $t$-Bu 3 P in x,y,z format in $\AA$.

Cy-Pip-phos

58

$15-0.86715353-0.21876755-0.23718132$

61.066342121 .304851411 .30759263

60.889775160 .161321680 .31752941

$61.826049450 .32486963-0.87301010$

$63.252967360 .53264862-0.42957082$

62.514224291 .523346191 .68720615

12.646919252 .414221292 .33063412

12.914939400 .643462242 .22877169

$13.942444560 .69550294-1.27961743$

$13.60962677-0.347190470 .14298774$

$11.17718565-0.786620380 .83069986$

$11.80110848-0.59174132-1.49387538$

$11.488721971 .13914299-1.54487443$

10.616700532 .241087200 .92074090

10.523272511 .082923772 .24761629

73.412976031 .710914610 .49417904

$63.089795592 .97352743-0.21502319$

64.822256571 .776966570 .95021969

$13.753420353 .07117057-1.09167922$

13.249486923 .824363710 .46960863

$12.039661412 .96297073-0.55097228$

15.482446191 .897180320 .07288378

15.081417080 .845276711 .48171353

14.949566842 .638877151 .62853885

$6-3.061482911 .16639566-1.06567132$

$6-1.691730741 .44552302-0.44033915$

$6-0.907814922 .37801170-1.35951567$

$6-1.674787403 .65912962-1.65608287$

$6-3.025266893 .35827827-2.28044581$

$6-3.832767492 .43644977-1.38472950$

$1-4.792139052 .17266440-1.86922383$

$1-4.091616152 .96709895-0.44335970$

$1-3.578006034 .29581118-2.48176646$

$1-2.868795632 .86688805-3.26507616$

$1-1.077722794 .31378746-2.31999898$

$1-1.828871374 .21938753-0.70831966$

$1-1.833956721 .945470090 .54519635$

$10.076038532 .64214945-0.91496181$

$1-0.692588871 .84909475-2.31513619$ 
$1-2.898316140 .59233749-2.00345135$

$1-3.662166360 .51140666-0.40723178$

$6-1.38590670-2.388151171 .40183640$

$6-1.46621728-0.863023641 .42169631$

$6-2.85301232-0.420638111 .86486387$

$6-3.16965723-0.983517473 .24292803$

$6-3.08918905-2.500183583 .25060821$

$6-1.73539388-2.984483242 .75754809$

$1-1.71875525-4.089779852 .69720984$

$1-0.95320404-2.698895223 .49421501$

$1-3.28902078-2.892772914 .26607990$

$1-3.88625956-2.909049992 .59377170$

$1-4.17154312-0.646373213 .56921721$

$1-2.44480085-0.571785033 .97898412$

$1-0.75425583-0.492935722 .19784975$

$1-2.935346840 .685119991 .87403822$

$1-3.61289358-0.794189871 .14506447$

$1-2.09172177-2.763802770 .62979537$

$1-0.37837917-2.726203681 .07987928$

(Cy-Pip-phos) $\mathrm{Pd}(0)$

59

$46-1.945340160 .627266352 .42726922$

$60.96228445-1.45142460-0.66158479$ $60.02852473-1.217895390 .51804155$

$6-1.21801305-2.085798740 .44271383$

$6-0.87120736-3.534943340 .23123282$

$61.23768806-2.92564273-0.86522436$

$11.84407341-3.11496544-1.77167654$

$11.78079283-3.344518660 .00566080$

$1-1.77020943-4.169754980 .11268393$

$1-0.27753139-3.928030731 .08190143$

$10.57173657-1.519385101 .44245529$

$1-1.77000189-1.972275971 .40208817$

$1-1.92282927-1.72470343-0.33078748$

$10.57761371-0.98941946-1.59240806$

$11.94329524-0.97023994-0.47821119$

$7-0.01924634-3.74287128-0.99733365$

$6-0.76619899-3.34913659-2.21819592$

$60.33910197-5.17729759-1.10452592$

$1-1.68733525-3.95296025-2.29055548$

$1-0.13122439-3.52470493-3.10356736$

$1-1.03892446-2.28120470-2.17094636$

$1-0.58503526-5.77659178-1.18474317$

$10.90251058-5.48546124-0.20685600$

$10.95970994-5.33536053-2.00374365$ 
$6-1.631367332 .68739367-0.30367315$

$6-0.877144221 .40699756-0.67461437$

$6-1.757082220 .56879675-1.59654045$

$6-2.190829751 .34792483-2.82995534$

$6-2.936918022 .61268783-2.44725990$

$6-2.094028953 .46945691-1.52070749$

$1-2.667056804 .35454512-1.18378210$

$1-1.212847473 .85992527-2.07374477$

$1-3.224029783 .18139529-3.35181355$

$1-3.881976602 .33696318-1.93206799$

$1-2.817191600 .70495468-3.47852778$

$1-1.291423921 .61869240-3.42433381$

$10.068148651 .66810632-1.20571637$

$1-1.23099709-0.35393178-1.92240489$

$1-2.657679080 .24657676-1.02621746$

$1-2.504755502 .379823680 .31729010$

$1-1.017660503 .328560830 .35502446$

61.649831060 .650153462 .72437692

61.242628221 .220065951 .36788332

61.244634632 .745959041 .38505423

62.553185703 .312489991 .91268444

62.869523052 .765885353 .29198837

62.944475411 .250294333 .24820209

13.159471750 .835846484 .25151491

13.792521950 .948196832 .59611034

13.815673113 .191413883 .67591071

12.071344613 .074593074 .00115490

12.495926624 .417903421 .92596352

13.379430293 .047828671 .21767402

11.967385170 .884343330 .58885521

11.052946213 .140189650 .36716855

10.408945803 .094615942 .03211045

10.818414690 .854044383 .43721676

$11.74302065-0.455241172 .67714858$

$15-0.428747890 .549860060 .91433769$

(Cy-Pip-phos) $)_{2} \mathrm{Pd}(0)$

117

$460.27229869-0.025484520 .30420104$ $63.29058719-2.14806485-2.80722308$

$62.35302234-1.90218484-1.63204002$

$61.10124326-2.76758003-1.70032120$

$61.44511330-4.21867037-1.91363883$

$63.54238272-3.62379479-3.03300142$

$14.12814045-3.80377173-3.95440531$

$14.09920740-4.06180239-2.18136048$ 
$10.54531497-4.85437918-2.01843786$ $12.04984093-4.61154985-1.07126307$ $12.89281201-2.20421672-0.70513499$ $10.55006522-2.65822339-0.74015355$ $10.39352575-2.40963769-2.47318625$ $12.92761326-1.66742063-3.73659348$ $14.27916718-1.68993127-2.60647082$ $72.27860093-4.43151903-3.15046811$ $61.51619136-4.03806448-4.36119175$ $62.63152671-5.86793709-3.25768328$ $10.58393645-4.62756538-4.40771961$ $12.12908196-4.23616076-5.25713205$ $11.26469779-2.96530461-4.31703997$ $11.70588195-6.46554232-3.32940674$ $13.20052910-6.17367506-2.36309648$ $13.24621916-6.02869606-4.16032410$ $60.656649471 .97268128-2.45586658$ $61.465805770 .72626895-2.83269787$ $60.63093859-0.13386558-3.77668071$ $60.188526380 .64288688-5.00684786$ $6-0.632627671 .85446680-4.61247158$ $60.154526352 .74205756-3.66643858$ $1-0.469280093 .59226847-3.32753921$ $11.014461993 .18627477-4.21095610$ $1-0.945868912 .41905904-5.51050091$ $1-1.561564561 .50983465-4.10830259$ $1-0.38432041-0.01690923-5.68644428$ $11.085661050 .97629559-5.57184649$ $12.403689861 .02772772-3.35287786$ 1 1.19762874 - $1.02996850-4.10277939$ $1-0.26767519-0.49579558-3.22602725$ $1-0.197767791 .62104309-1.82626915$ $11.254046082 .63441467-1.80011165$ $63.90866852-0.011646190 .64338601$ $63.557054760 .53044945-0.74126375$ $63.562529092 .05444121-0.72314811$ $64.887308602 .60135746-0.21524864$ 65.219764232 .058131221 .16194892 65.229323860 .540918351 .15330005 15.431252960 .147368672 .16781116 16.056523320 .183619890 .50355512 16.193139082 .450637101 .51177502 14.453286652 .409577611 .88596797 $14.850096233 .70690107-0.20047474$ $15.697213172 .32370305-0.92375529$ $14.318048480 .19144940-1.48235452$ 
$13.350628852 .45950317-1.73252380$ $12.738846542 .39507055-0.05430867$ 13.081469060 .271669091 .33580780 $13.94212770-1.120485780 .64318496$ $151.90338266-0.13468492-1.24228966$ $6-2.984440572 .127800703 .14738917$ $6-1.738539701 .861258752 .31223869$ $6-1.629909752 .802435161 .11904657$ $6-1.790663964 .240953451 .53685963$ $6-3.146788843 .597378253 .47299385$ $1-4.109853273 .801327473 .97851634$ $1-2.330405243 .944653034 .13636112$ $1-1.766595014 .935576920 .67562360$ $1-0.991686344 .540446762 .24478865$ $1-0.852690042 .068173172 .95573664$ $1-0.628224552 .665530920 .65512818$ $1-2.347844362 .538475510 .31805420$ $1-3.899830101 .730575922 .66705084$ $1-2.909803631 .599627384 .11837959$ $7-3.090067394 .483906752 .25881362$ $6-4.239132884 .215458871 .35899770$ $6-3.147462845 .900897982 .69243646$ $1-4.155888564 .860051160 .46668819$ $1-5.178997994 .435869221 .89358425$ $1-4.233695033 .158552171 .04472232$ $1-3.090569736 .555476671 .80509377$ $1-2.297575956 .114531043 .36288977$ $1-4.095886716 .083602913 .22683096$ $6-2.62262177-1.845092890 .30424267$ $6-3.00414634-0.625402991 .15054786$ $6-3.805847880 .340245400 .28291750$ $6-5.02994633-0.32916108-0.32276863$ $6-4.63035107-1.51818109-1.17367387$ $6-3.82106018-2.50724435-0.35521981$ $1-3.47356319-3.34229326-0.99438053$ $1-4.47303867-2.961070540 .42102134$ $1-5.52537060-2.00386596-1.60478389$ $1-4.01641989-1.15737581-2.02740288$ $1-5.607543950 .40415248-0.91784000$ $1-5.70149708-0.671157300 .49393186$ $1-3.63240695-0.945672572 .01286030$ $1-4.132419591 .223776940 .86876798$ $1-3.144504790 .71301013-0.53296226$ $1-1.89513874-1.48558462-0.46460661$ $1-2.06541109-2.579269170 .91723275$ $60.26426855-0.257927753 .94058132$ 
$6-1.11253452-0.688025473 .43749452$

$6-1.18651807-2.208044533 .34911180$

$6-0.83000797-2.862437494 .67470026$

$60.54166168-2.429147965 .15650320$

$60.62213695-0.917718055 .26177788$

$11.63622332-0.602833095 .57313824$

$1-0.07527433-0.568425716 .05276680$

$10.77830166-2.900559666 .12909603$

$11.30905175-2.781353244 .43394041$

$1-0.87800604-3.962589264 .56875849$

$1-1.58977878-2.587811235 .43783617$

$1-1.89755666-0.345027604 .15117407$

$1-2.19568133-2.532952553 .02761817$

$1-0.47029540-2.543006182 .56365609$

$11.00931680-0.536634923 .15884709$

10.327283590 .844947344 .04319811

$15-1.415267940 .101629851 .79006112$

$t$-Bu-Pip-phos

50

$15-1.50331628-0.49365744-0.07556121$

$6-1.771472931 .29208934-2.12615252$

$6-2.242962841 .14111793-0.68018019$

$6-3.750786540 .89593989-0.75159431$

$6-1.978607182 .418969390 .09811816$

$6-1.92407227-0.805849791 .74347317$

$6-1.403923630 .198736382 .75819898$

$6-3.43506145-0.935863441 .88653135$

$6-1.31347132-2.178036692 .03051782$

$1-1.59067869-2.496658333 .05541253$

$1-0.20537874-2.172163491 .98833013$

$1-0.303288190 .323842592 .72502303$

$1-1.64853573-0.154430523 .78154016$

$1-1.864889861 .195032122 .63914537$

$1-2.342426542 .358154771 .13969350$

$1-2.517467743 .26168299-0.38278994$

$1-0.909342232 .699112890 .12359530$

$1-0.706882601 .57544124-2.21227908$

$1-1.938477160 .36417729-2.70634675$

$1-2.350087172 .10194349-2.61473274$

$1-3.98995662-0.09744326-1.17944515$

$1-4.211183071 .66210794-1.40790021$

$1-4.241268160 .975592670 .23294748$

$1-3.88098955-1.553220511 .08309472$

$1-3.67079568-1.421831372 .85492373$

$1-3.938690190 .046651931 .88990211$ 
$1-1.68631959-2.944374561 .32355726$

$61.102865100 .92070752-0.35696656$

$60.38225758-0.338610290 .09303576$

$60.97561920-1.55106068-0.61589360$

$62.47529912-1.62862360-0.46050835$

$62.605576040 .80201918-0.22931698$

$13.118563411 .69575500-0.63318795$

12.906130550 .681658510 .83091509

$12.91440225-2.48594069-1.00594187$

$12.75059557-1.720914130 .60958439$

$10.59011859-0.450883571 .18021202$

$10.54931712-2.48095846-0.19135027$

$10.67179328-1.56700563-1.68248224$

$10.832439301 .20101547-1.39275146$

10.804445331 .784120080 .26668537

$73.16796994-0.38886198-0.95722663$

$62.97700930-0.24288146-2.42275071$

$64.61997890-0.49040055-0.67535305$

$13.33603215-1.15695608-2.92645121$

$13.549878360 .63149846-2.77752209$

$11.90913463-0.10047676-2.64945436$

$15.04081726-1.34956217-1.22608542$

$14.77551174-0.632308420 .40761504$

$15.122703550 .43695480-1.00051272$

( $t$-Bu-Pip-phos) $\operatorname{Pd}(0)$

51

$46-0.61617953-2.751120091 .05546784$

$61.88222528-0.879904572 .38229728$

$62.17466354-0.886487840 .88324881$

$63.09560156-2.079046490 .63161159$

62.868727680 .417398570 .45696205

$60.90562892-1.69787371-1.80654132$

$61.93545628-0.77491981-2.44164658$

$61.34969127-3.15463424-1.89541948$

$6-0.40024281-1.59025931-2.58509517$

$1-0.22440247-1.95235884-3.61791348$

$1-0.76638061-0.54873228-2.67456889$

$11.630306720 .29035190-2.40298796$

$12.04200673-1.03485954-3.51440644$

$12.93588424-0.86388808-1.98118126$

$12.493669990 .84524977-0.49091044$

13.952507260 .243201640 .31045610

12.785394431 .191956161 .24238634

$11.25478947-0.027535142 .70088792$

$11.35450613-1.811292652 .67596316$ 
$12.83964109-0.814882582 .93851113$ $12.58206296-3.038646460 .84111208$ $13.96580410-2.002959491 .31411636$ $13.49117208-2.10151172-0.39943126$ $10.60364157-3.81746292-1.41289735$ $11.43346000-3.43949962-2.96410680$ $12.32586479-3.34567976-1.42173505$ $1-1.18855596-2.22735524-2.13568377$ $6-0.193657931 .346144200 .93818402$ $6-0.414289210 .33925441-0.18154480$ $6-1.90076494-0.00372974-0.24476774$ $6-2.789018631 .20986736-0.32966316$ $6-1.095062972 .549634700 .79997241$ $1-0.949412883 .275540111 .62327909$ $1-0.905922893 .07483959-0.15770675$ $1-3.859207630 .92975032-0.29369962$ $1-2.614800451 .77536595-1.26717448$ $1-0.098942020 .80361515-1.14418757$ $1-2.12197757-0.62324780-1.13153517$ $1-2.14533854-0.672430460 .61556762$ $1-0.335917740 .861826721 .92577374$ 10.845479131 .724676010 .93258965 $7-2.550189972 .181491140 .79121304$ $6-2.941671131 .574701552 .08901000$ $6-3.362844473 .399106740 .57075870$ $1-4.028572561 .383238432 .07736444$ $1-2.690141682 .276097772 .90341854$ $1-2.405278440 .623677972 .23942113$ $1-4.429263113 .117805960 .51576138$ $1-3.059123753 .87919807-0.37543288$ $1-3.205312014 .099503991 .40989852$ $150.54430646-1.273187040 .00501241$

( $t$-Bu-Pip-pho $)_{2} \mathrm{Pd}(0)$

101

$46-0.19416609-0.05605376-0.17701188$ 62.350785491 .956484321 .36707914 $62.691875461 .93173063-0.12199572$ $63.589273930 .71316212-0.33367798$ $63.445412403 .20982218-0.52465659$ $61.488472221 .24706149-2.86634254$ $62.544676302 .16436100-3.46139669$ $61.91669512-0.21480903-2.98076701$ $60.192656671 .38605833-3.65922618$ $10.383147931 .09577239-4.71221828$ $1-0.191434442 .42407441-3.68523264$ 
$12.255970483 .23350382-3.41010356$ $12.672227381 .92387283-4.53604031$ $13.535041332 .05160522-2.98269939$ $13.079198363 .68055129-1.45513189$ $14.517520902 .99272370-0.69385058$ 13.410480503 .966212030 .28127334 11.683066842 .789536001 .64766157 11.855829831 .012262701 .67287469 13.284975292 .081765651 .95112193 $13.02494717-0.23079860-0.17668092$ 14.416769500 .751644730 .40371794 $14.049937250 .69264466-1.33800113$ $11.17162383-0.86817890-2.47549438$ $11.97156549-0.49498370-4.05268860$ $12.90726757-0.40870041-2.53524089$ $1-0.591726180 .71138883-3.25949454$ $60.422319474 .25028133-0.06719892$ $60.173250713 .24573421-1.18228233$ $6-1.326206332 .95771074-1.22739863$ $6-2.160300974 .20975447-1.35410559$ $6-0.405281965 .49997425-0.25027776$ $1-0.229260536 .230474470 .56203556$ $1-0.167815545 .99453545-1.21367276$ $1-3.243216993 .98563027-1.30852783$ $1-1.956524494 .72374821-2.31526399$ $10.491613983 .71602821-2.14104152$ $1-1.581783292 .31717038-2.09183145$ $1-1.601304772 .33790302-0.34303299$ 10.216719213 .799457550 .92424470 $11.479581594 .57098007-0.04364710$ $7-1.880763175 .22164297-0.27764520$ $6-2.340834144 .717551231 .04117000$ $6-2.605013856 .47954416-0.58491576$ $1-3.436450244 .587912081 .01470125$ $1-2.073512085 .450968271 .82174623$ $1-1.863987803 .749033211 .26240063$ $1-3.689012056 .27545881-0.63603586$ $1-2.256810196 .87392950-1.55497384$ $1-2.404839757 .220581530 .20951998$ $151.080340741 .60030317-1.04991388$ $6-1.25738597-0.460746652 .98369813$ $6-2.22629476-1.388084652 .25388479$ $6-3.45165396-0.555559401 .88295865$ $6-2.66005969-2.528382063 .17356539$ $6-2.62612700-2.60046411-0.57505631$ $6-3.47948766-3.689824580 .05494122$ 
$6-3.51093078-1.50893223-1.17037749$

$6-1.82469499-3.18637156-1.73492455$

$1-2.53145647-3.53004265-2.51657701$

$1-1.21915150-4.06805086-1.44784975$

$1-2.87241840-4.505589960 .49657521$

$1-4.11983871-4.15356779-0.72327393$

$1-4.15200853-3.299067500 .84042072$

$1-2.97788572-3.439162022 .63495874$

$1-3.52407289-2.198368073 .78495622$

$1-1.86773443-2.800713303 .89292765$

$1-0.31208259-0.962424223 .26579070$

$1-0.987643420 .401398572 .33556342$

$1-1.73238337-0.087687543 .91486597$

$1-3.220117810 .187302371 .09283388$

$1-3.78747892-0.005566542 .78560424$

$1-4.29890060-1.182698971 .54946864$

$1-2.89299965-0.68481791-1.58146369$

$1-4.10284138-1.94356704-2.00156379$

$1-4.22559881-1.08387506-0.44763750$

$1-1.16491342-2.41955781-2.19204760$

$60.32457048-3.509573942 .28585625$

$6-0.22363396-3.298579930 .88314712$

$60.95881504-3.14251399-0.07064154$

$61.91413915-4.306690220 .00486193$

$61.27240252-4.686110022 .33478642$

$11.69148338-4.839624883 .34800220$

$10.74764466-5.615646842 .03646493$

$12.79595399-4.16420841-0.64898252$

$11.41014969-5.24564409-0.30197555$

$1-0.80363399-4.210207460 .61197668$

$10.62116152-3.07447195-1.12181151$

$11.44788063-2.157009840 .11590876$

$10.81360495-2.590537312 .66917896$

$1-0.48854819-3.743592262 .99599290$

$72.43567538-4.550068861 .39408362$

$63.31765199-3.434472081 .81904197$

$63.21827435-5.810882091 .40420401$

$14.18708706-3.386122471 .14067936$

$13.66000819-3.619467022 .85217810$

$12.76636839-2.480836631 .77555954$

$14.06791782-5.721019740 .70451784$

$12.56891274-6.645713331 .08846331$

$13.59551764-6.002165322 .42429900$

$15-1.37623715-1.829440360 .62212563$

t-Bu-Amphos 
46

$15-0.42965454-0.44067252-0.49386775$

$61.44301724-0.30112949-0.35663837$

$62.03514576-1.62497878-0.75648779$

$6-0.179779771 .29095852-2.57346559$

$6-0.856549621 .25265205-1.20515180$

$6-2.364413021 .26145649-1.43763006$

$6-0.436071782 .45990849-0.38576090$

$6-0.88654476-0.573129531 .33365166$

$6-0.287371010 .439704212 .29533529$

$6-2.40692806-0.534313441 .43522465$

$6-0.40651268-1.968076351 .72761619$

$1-0.75183380-2.202326772 .75478292$

$10.70134622-2.034539221 .74634624$

$11.59736335-1.98389030-1.70916164$

$11.82068086-2.395184280 .01073104$

10.819666150 .401465982 .33581066

$1-0.641706710 .213281393 .32228470$

$1-0.584991991 .477005722 .06600714$

$1-1.060939432 .584607120 .51698029$

$1-0.556132503 .38350248-0.98860490$

$10.624000432 .41165066-0.06572108$

$10.909870271 .48290467-2.51277232$

$1-0.347196910 .35576111-3.14253092$

$1-0.608224452 .12216973-3.16816425$

$1-2.680888650 .41728058-2.07928205$

$1-2.653887752 .20226860-1.94844842$

$1-2.940026041 .20761597-0.49707270$

$1-2.89681768-1.154966470 .65983325$

$1-2.72308111-0.920349062 .42500901$

$1-2.797442910 .496605461 .35290229$

$1-0.79923493-2.751030451 .05080962$

$11.71994102-0.011246520 .67487234$

$11.754686830 .51041770-1.04205549$

$73.54122496-1.63819945-0.95682585$

$64.23243666-1.049300310 .21495129$

$63.89012885-0.87365943-2.17919612$

$63.97771287-3.04729509-1.12598407$

$13.38833642-1.33351231-3.04763651$

$14.98340702-0.89192969-2.32634664$

$13.551682000 .17023599-2.07198620$

$13.43076634-3.49815965-1.97201455$

$13.75583172-3.60998583-0.20296539$

$15.06330490-3.07663488-1.32568014$

$15.32352209-1.150037290 .08280879$

$13.91862607-1.581096891 .13033676$ 
( $t$-Bu-Amphos) $\operatorname{Pd}(0)$

47

$46-2.258554461 .185954691 .31952453$ $60.09897815-0.70881164-0.09765673$ $6-0.26269719-1.439017891 .16618764$ $6-2.306094650 .64465040-2.04230189$ $6-0.975976591 .35156560-1.80865073$ $6-1.171160702 .84936762-1.99544239$ $60.059412880 .81956750-2.79021955$ 60.910372202 .029705520 .58390939 $62.023179292 .18135405-0.44022578$ 60.397518813 .398193601 .01240432 61.441164021 .305651431 .81436729 12.199811701 .942036512 .31201863 11.942968960 .351283581 .55287111 $1-1.34704781-1.273259521 .37225711$ $10.29218197-1.041116602 .03734207$ $12.409648661 .21081579-0.81145388$ 12.874611382 .710422040 .03455605 $11.714393142 .79115224-1.31016195$ $11.061364411 .26060963-2.64924502$ $1-0.267109481 .06760740-3.82032537$ $10.15492834-0.28309226-2.75293899$ $1-2.24630713-0.44490030-1.84413886$ $1-3.093838931 .05545318-1.38034952$ $1-2.614967580 .77220839-3.09940004$ $1-1.866860273 .26306129-1.23858738$ $1-1.608788613 .04179883-2.99609423$ $1-0.217105173 .40768099-1.94245565$ $1-0.387389663 .295525791 .78828609$ 11.236426123 .989782091 .43141210 $1-0.033727233 .968456980 .17060807$ 10.628797111 .116613982 .54630303 $11.18770063-0.68824303-0.30974799$ $1-0.42052498-1.14905679-0.97090316$ $7-0.00305056-2.930744171 .14965761$ $61.45274293-3.198614361 .05016232$ $6-0.71851021-3.569349050 .01856403$ $6-0.51984197-3.488905432 .42481804$ $1-1.78469539-3.285197970 .06292483$ $1-0.61703956-4.665294170 .09431196$ $1-0.28826684-3.22801471-0.93699825$ $1-1.60844827-3.317281012 .47692037$ $1-0.02893688-2.976883893 .26959848$ 
$1-0.30745661-4.570952892 .46902704$ $11.63056374-4.288012501 .04712296$ $11.96241820-2.741928581 .91663933$ $11.84423327-2.757092710 .11840969$ $15-0.569028261 .03523111-0.00291364$

$(t \text {-Bu-Amphos })_{2} \operatorname{Pd}(0)$

93

$46-0.08475558-0.07625561-0.06164121$ $62.32870746-1.97925317-1.64629877$ $61.99754107-2.75404859-0.39822820$ $6-0.10175275-0.60043019-3.51885557$ $61.227798340 .11255115-3.29387546$ $61.010363941 .61127365-3.45031929$ $62.24684143-0.37901148-4.31131506$ $63.163504600 .75271982-0.93657398$ $64.290825840 .86099964-1.94918835$ $62.660970212 .13849425-0.54289949$ 63.671854730 .060120580 .32325816 14.440806870 .697962280 .80425698 $14.16351652-0.908351480 .09941673$ $10.91185182-2.64125609-0.17583516$ $12.54848218-2.359337090 .47747394$ $14.64080524-0.12574811-2.31093836$ $15.163482671 .35485530-1.47617364$ $14.014910701 .47275007-2.82754517$ $13.238314870 .09031003-4.19398785$ $11.88578427-0.13067766-5.32945633$ $12.37735343-1.47775447-4.28973103$ $1-0.02913087-1.69726145-3.37163520$ $1-0.87834972-0.22112098-2.82428956$ $1-0.43856651-0.43610513-4.56232405$ $10.346410752 .00304198-2.65257931$ $10.523811461 .81202567-4.42590809$ $11.960751182 .17737651-3.43790746$ 11.833613282 .044878720 .19363762 $13.488079792 .71770620-0.08432667$ $12.290645122 .70919752-1.41410768$ $12.85291839-0.097416161 .05643094$ $13.41236401-1.94621968-1.87915051$ $11.80313134-2.40261412-2.52453756$ $72.29997563-4.23810339-0.44551185$ $63.76099157-4.47461128-0.57354754$ $61.59255755-4.88568163-1.57889712$ $61.81906545-4.832735540 .82943970$ $10.52009815-4.62597847-1.53674459$ 
$11.71405149-5.98045349-1.50560522$

$12.02033448-4.53772497-2.53353882$

$10.72524321-4.702011590 .89998460$

$12.30636907-4.319617751 .67595041$

$12.06869125-5.907776830 .85666776$

$13.95548964-5.56126690-0.59423196$

$14.28238344-4.023425580 .28881669$

$14.12632275-4.02003431-1.50963902$

$151.66652226-0.23697492-1.49820113$

$6-2.500942231 .916940571 .29085720$

$6-1.344094992 .855139971 .06558836$

$6-3.34864926-0.60769576-0.85644835$

$6-3.41480160-0.752553280 .66100293$

$6-3.28735256-2.230844741 .00370061$

$6-4.74153137-0.205116441 .16774917$

$6-1.60041356-0.196484393 .12571168$

$6-2.85989499-0.178513543 .97679496$

$6-0.89823556-1.545186043 .23907423$

$6-0.632476810 .873045743 .62106347$

$1-0.288861660 .607157834 .64089537$

$1-1.110904571 .869586113 .70407796$

$1-0.775915562 .530297040 .16266537$

$1-0.635784152 .828502181 .91475821$

$1-3.426702980 .769286813 .88682747$

$1-2.58448696-0.283343295 .04580545$

$1-3.54415131-1.013064983 .73830390$

$1-4.82974339-0.223947002 .26778841$

$1-5.56545830-0.826784910 .76236606$

$1-4.935830120 .827746210 .82077610$

$1-3.372176650 .44954994-1.18916643$

$1-2.41581798-1.05879402-1.25394177$

$1-4.22072506-1.11732364-1.31479859$

$1-2.28192592-2.618369340 .74318928$

$1-4.03196335-2.804230930 .41540757$

$1-3.48606539-2.439819572 .07160115$

$10.02655791-1.553859112 .62447119$

$1-0.61891919-1.723865154 .29749966$

$1-1.54137790-2.383538482 .91777253$

10.262647930 .943666642 .96800137

$1-3.062801122 .119319682 .22584009$

$1-3.214254141 .962086800 .44441250$

$7-1.709989074 .314233780 .88788909$

$6-2.362287284 .844027522 .11322951$

$6-2.615069634 .49442530-0.27451780$

$6-0.452758435 .067304130 .64120609$

$1-2.149831534 .04591513-1.16994548$ 
$1-2.781296495 .57291222-0.43960026$

$1-3.583205704 .00599766-0.07532276$

$10.023963044 .68164301-0.27670750$

10.231025804 .927945611 .49638343

$1-0.680619246 .141179560 .52201319$

$1-2.580920935 .918119431 .97947407$

$1-1.684117444 .710374362 .97473717$

$1-3.304053784 .299721242 .29260993$

$15-1.888761520 .150220831 .29912663$

$t-\mathrm{Bu}_{3} \mathrm{P}$

40

$150.057945040 .01447655-0.83584541$

$61.77265036-0.047774050 .00476696$

61.843413950 .295307841 .48601794

$62.36795616-1.43737745-0.20351972$

$62.676521300 .90145588-0.78307116$

$6-0.835551921 .56175745-0.15108377$

$6-1.344556571 .501920941 .28259075$

$60.096993122 .76248837-0.28596002$

$6-2.003241061 .84523952-1.09657669$

$6-0.91303098-1.50753021-0.21195194$

$6-0.77174383-1.872913001 .25929224$

$6-2.39328313-1.30883420-0.52394938$

$6-0.47515145-2.68917990-1.07967997$

$12.630199910 .68267399-1.86713314$

$13.725865360 .76265091-0.44800878$

$12.418002841 .96331656-0.63978839$

$12.33137965-1.74498630-1.26689184$

$11.88181353-2.215904240 .41149175$

$13.43495035-1.405480270 .09912738$

11.578071121 .349399921 .68722665

12.886226420 .154234931 .84352589

$11.18900836-0.348416212 .10118747$

$1-1.661681411 .88584578-2.14858961$

$1-2.444459442 .83134842-0.84008664$

$1-2.807150361 .09433675-1.02756000$

$10.549454032 .82418013-1.29466963$

10.904146312 .770596270 .46853188

$1-0.497979703 .68618965-0.13064995$

$1-2.148692130 .754094421 .41092229$

$1-1.775919082 .488543031 .55884814$

$1-0.541405561 .273887752 .00701356$

$1-0.62671494-2.46776056-2.15350962$

$1-1.09333265-3.57369256-0.81917453$

$10.58142817-2.96873522-0.93891555$ 
$1-2.55428243-0.98922908-1.57176960$

$1-2.88477921-0.584563080 .15087710$

$1-2.91026640-2.28204489-0.39060208$

$10.25415242-2.192940001 .51910508$

$1-1.44431579-2.726912261 .48942101$

$1-1.05262899-1.039676311 .92904007$

$\left(t-\mathrm{Bu}_{3} \mathrm{P}\right) \mathrm{Pd}(0)$

41

$150.051024460 .01202439-0.72804882$ $61.77902451-0.047975610 .07695116$ 61.833024410 .296024391 .55895118 $62.36802450-1.43897562-0.13004883$ $62.682024390 .90402437-0.70604881$ $6-0.845975551 .56902439-0.08104884$ $6-1.348975511 .495024391 .35595111$ $60.091024462 .76402433-0.21404884$ $6-2.015975571 .84902437-1.02204879$ $6-0.92397556-1.51797562-0.14104884$ $6-0.77497551-1.870975661 .33295111$ $6-2.40197548-1.30997555-0.45204883$ $6-0.48297554-2.69997566-1.00404878$ $12.627024560 .68202437-1.79204889$ $13.729024560 .76602436-0.36504884$ $12.418024451 .96502442-0.57004883$ $12.32002440-1.73997562-1.19604882$ $11.87902441-2.215975690 .48595117$ $13.43602457-1.410975620 .16795116$ 11.566024451 .350024411 .75895111 12.875024470 .153024391 .91695122 $11.17902449-0.351975612 .17095117$ $1-1.664975501 .88402433-2.07404872$ $1-2.456975552 .83302433-0.75904884$ $1-2.814975591 .09302444-0.96104886$ $10.538024452 .81602437-1.22604879$ 10.901024492 .765024490 .53795115 $1-0.499975533 .68902428-0.05604884$ $1-2.151975480 .746024381 .48395113$ $1-1.777975532 .481024341 .63195113$ $1-0.542975521 .269024442 .07695107$ $1-0.62997553-2.46697562-2.07904883$ $1-1.10297558-3.58297556-0.74204882$ $10.57502445-2.97397559-0.86704882$ $1-2.54997549-0.98697560-1.50204881$ $1-2.88997564-0.583975600 .22295117$ $1-2.92297563-2.28097551-0.31904884$ 
$10.25102445-2.189975671 .59095113$

$1-1.44697556-2.724975521 .56295113$

$1-1.05797550-1.034975581 .99995116$

$460.207024470 .04902439-2.94604893$

$\left(t-\mathrm{Bu}_{3} \mathrm{P}\right) \mathrm{Pd}(0)$

81

$460.005024720 .00401244-0.00402465$

$150.002024722 .27901249-0.00402465$

$6-0.461975292 .966012521 .71397534$

$6-0.156975284 .438012641 .95497539$

$6-1.951975302 .729012531 .93797531$

60.255024722 .111012502 .75797525

$61.717024732 .97901253-0.46002466$

$61.760024724 .45101266-0.85102466$

62.659024642 .758012340 .71797534

$62.271024632 .12701254-1.60202465$

$6-1.253975222 .97501235-1.26002464$

$6-1.618975234 .44501261-1.10102460$

$6-0.702975282 .75601248-2.66502461$

$6-2.516975242 .11901240-1.17302463$

10.049024721 .035012412 .57597532

$1-0.123975272 .387012533 .76397529$

11.349024702 .245012332 .75597539

$1-2.229975291 .682012481 .70497539$

$1-2.590975363 .413012551 .34897533$

$1-2.175975392 .913012553 .00897541$

10.927024714 .649012611 .96097531

$1-0.548975304 .727012682 .95297542$

$1-0.632975295 .098012491 .20697537$

$12.229024821 .05001239-1.33202467$

$13.329024722 .41201238-1.77702460$

$11.722024732 .25301246-2.54802475$

12.613024641 .712012451 .07897534

12.459024843 .442012351 .56397536

13.696024822 .956012530 .37597534

$11.220024754 .65401225-1.79202471$

$12.818024804 .74701266-1.01402471$

$11.348024775 .11001258-0.06602465$

$1-2.259975271 .04401247-1.27402470$

$1-3.196975302 .40601234-2.00202474$

$1-3.064975332 .24201254-0.22502465$

$1-0.365975271 .70901243-2.80302462$

$10.128024733 .44001250-2.91602454$

$1-1.518975332 .95201234-3.39202461$

$1-2.164975244 .64401250-0.16102465$ 
$1-2.290975164 .74001221-1.93502459$

$1-0.735975285 .10801224-1.13602469$

$150.00202472-2.274987650 .00197535$

$6-0.03697528-2.971987441 .77797535$

$6-0.40297527-4.443987331 .91597536$

$61.33002477-2.746987542 .41497531$

$6-1.01597531-2.119987442 .58497539$

$6-1.51897533-2.97098751-0.91902468$

$6-1.45397527-4.44198747-1.30902467$

$6-2.75197537-2.75098749-0.04902465$

$6-1.73197527-2.11498757-2.16502461$

$61.55702477-2.97298761-0.85202465$

$61.86202471-4.44298740-0.60002468$

$61.42102473-2.75398751-2.35502467$

$62.74502461-2.11698766-0.41302464$

$1-0.76297529-1.043987592 .48097530$

$1-0.93697525-2.405987463 .65497532$

$1-2.06497533-2.246987542 .27397528$

$11.66802471-1.699987612 .28297534$

$12.10602467-3.429987622 .02297535$

$11.24202471-2.940987543 .50397530$

$1-1.44697530-4.649987651 .61797538$

$1-0.30597527-4.738987402 .98197546$

$10.26002472-5.102987721 .32697537$

$1-1.77397532-1.04098757-1.88602469$

$1-2.69497521-2.40298767-2.63502464$

$1-0.93497528-2.23198767-2.91802463$

$1-2.80497534-1.706987580 .31697535$

$1-2.79797537-3.440987540 .81397536$

$1-3.65197523-2.94198747-0.66802463$

$1-0.67497529-4.64698739-2.06602464$

$1-2.42597539-4.73798747-1.75702462$

$1-1.27397532-5.10198779-0.44102467$

$12.52602475-1.04198761-0.58602468$

$13.63202469-2.40498753-1.01502464$ $13.00002472-2.236987550 .65197536$

$11.13202469-1.70898755-2.58502469$

$10.69502472-3.44198747-2.82502470$

$12.40702479-2.94698758-2.82602463$

$12.13302462-4.644987540 .45197534$

$12.73402469-4.73898740-1.22202465$

1 1.02102475-5.10498758 -0.87302468 


\section{NMR Spectra}

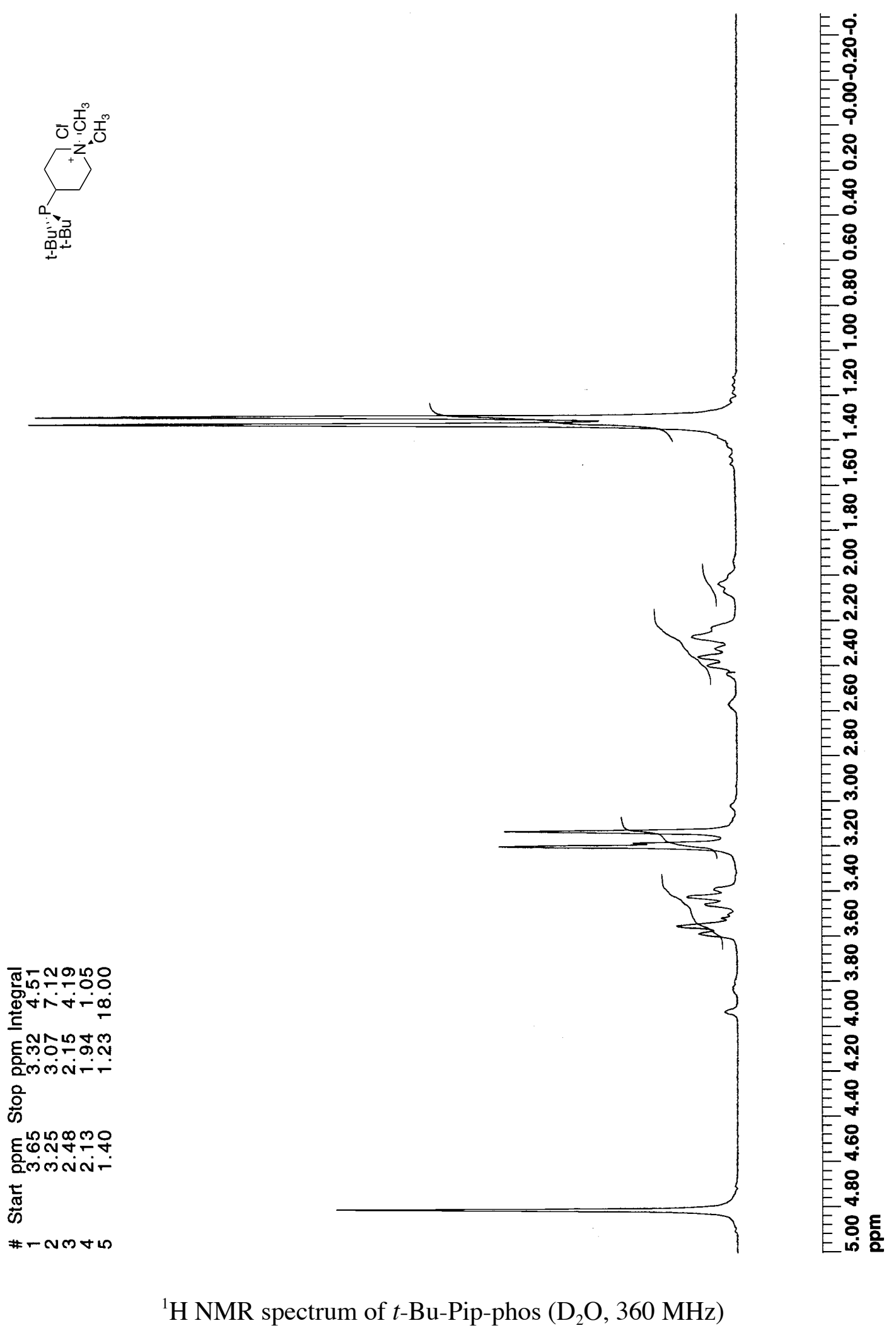




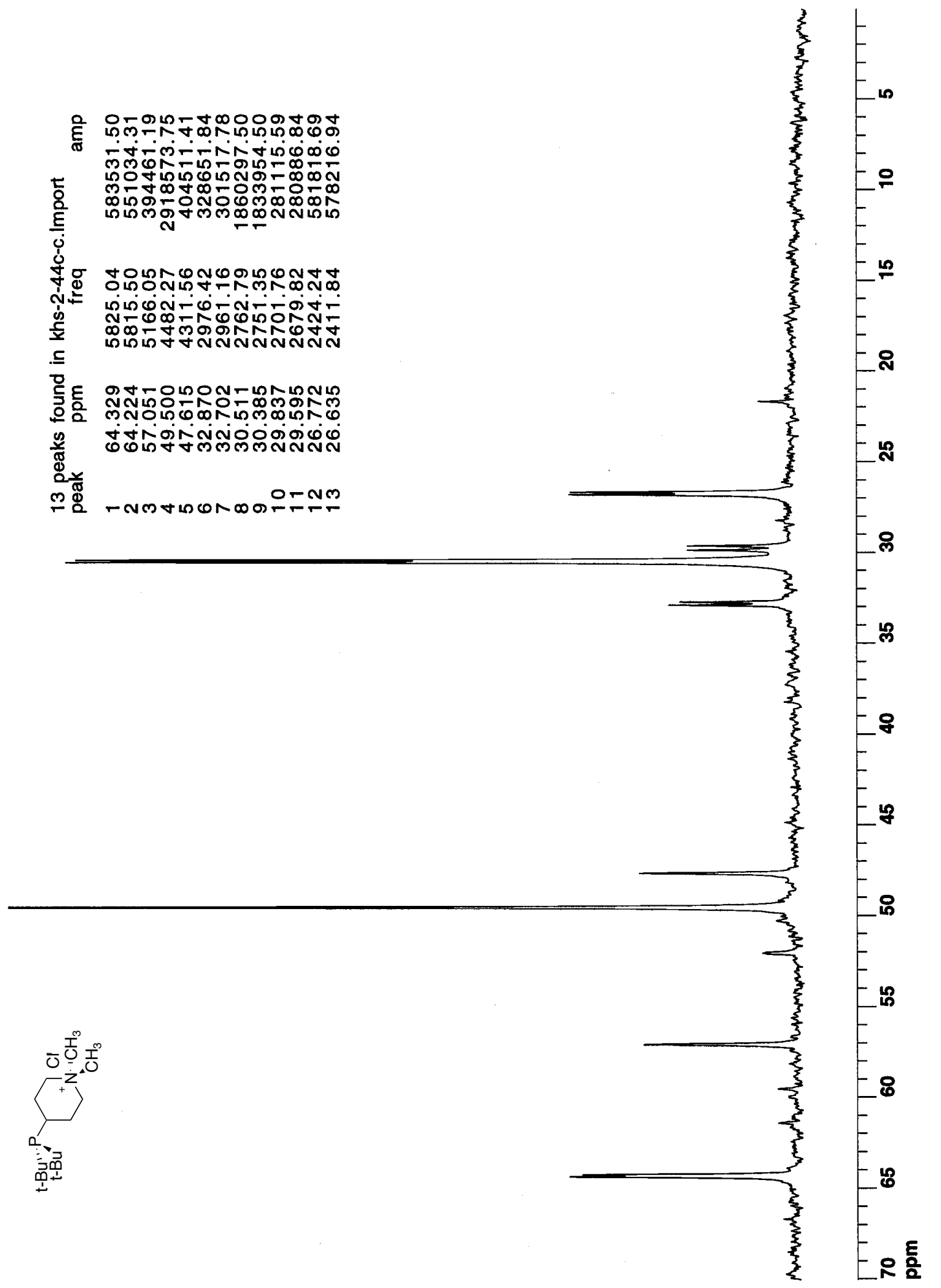

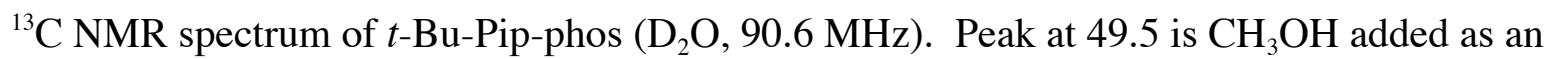
internal standard. 


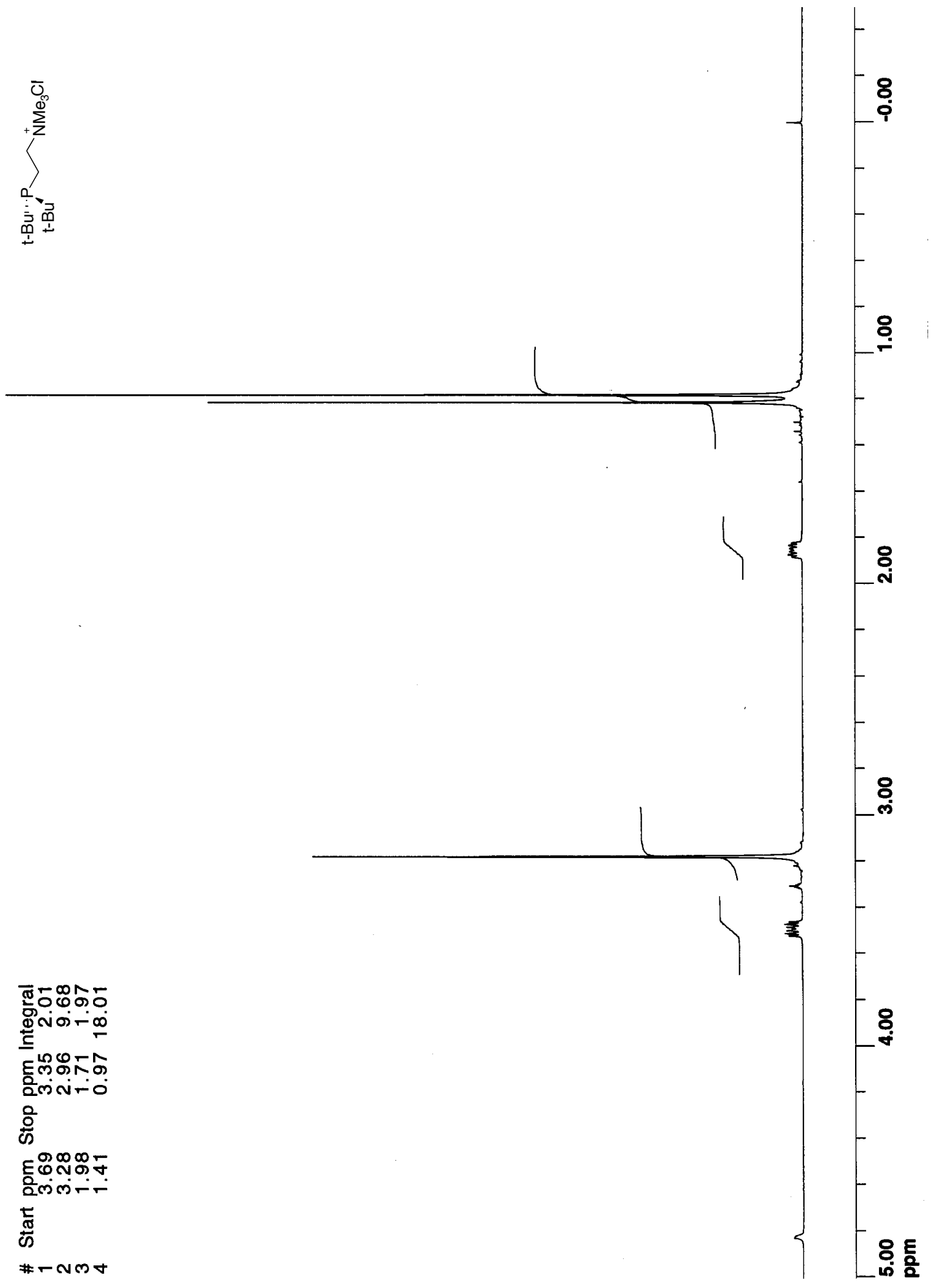

${ }^{1} \mathrm{H}$ NMR spectrum of $t$-Bu-Amphos $\left(\mathrm{CD}_{3} \mathrm{OD}, 360 \mathrm{MHz}\right)$. 


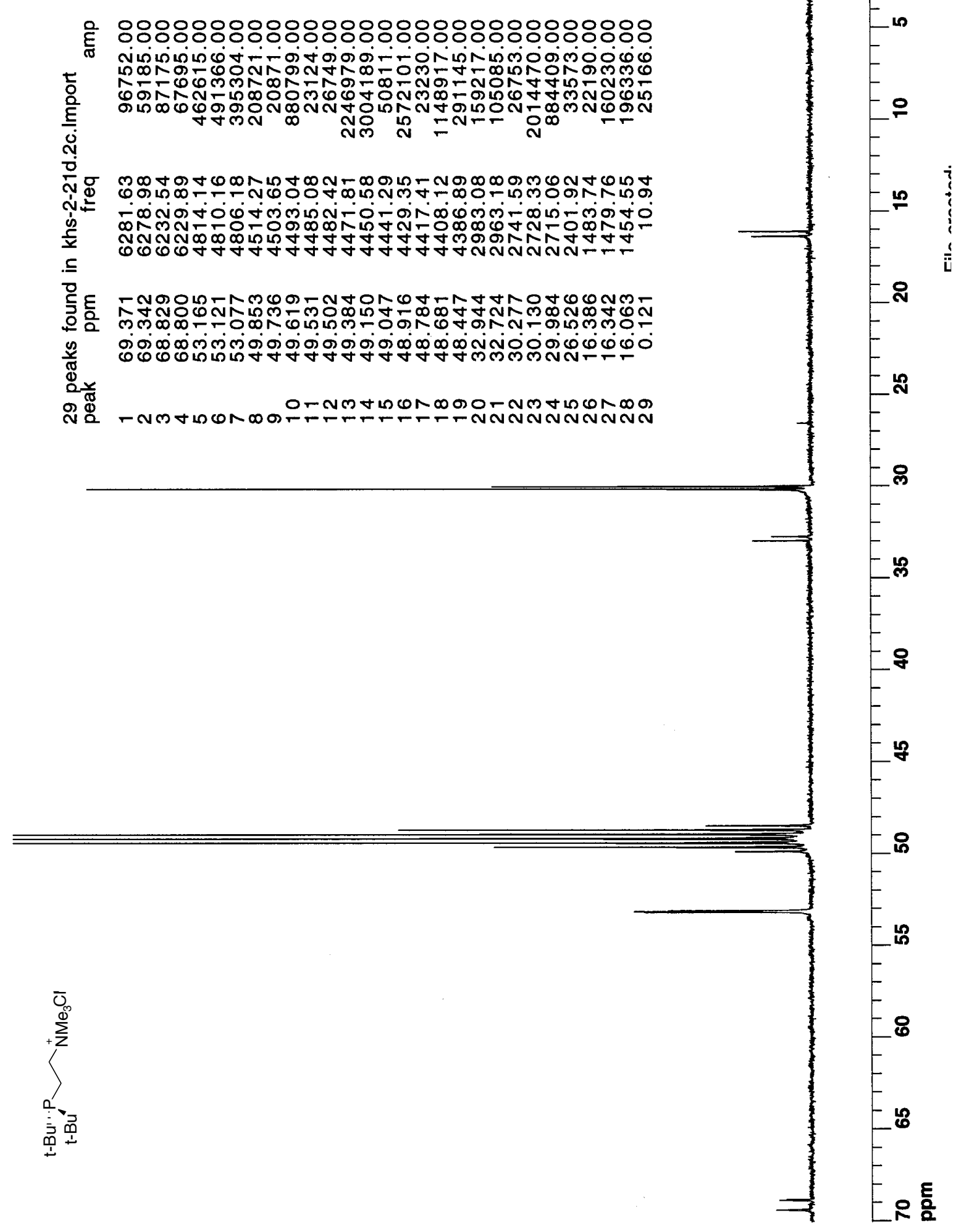

${ }^{13} \mathrm{C}$ NMR spectrum of $t$-Bu-Amphos $\left(\mathrm{CD}_{3} \mathrm{OD}, 90.6 \mathrm{MHz}\right)$. 

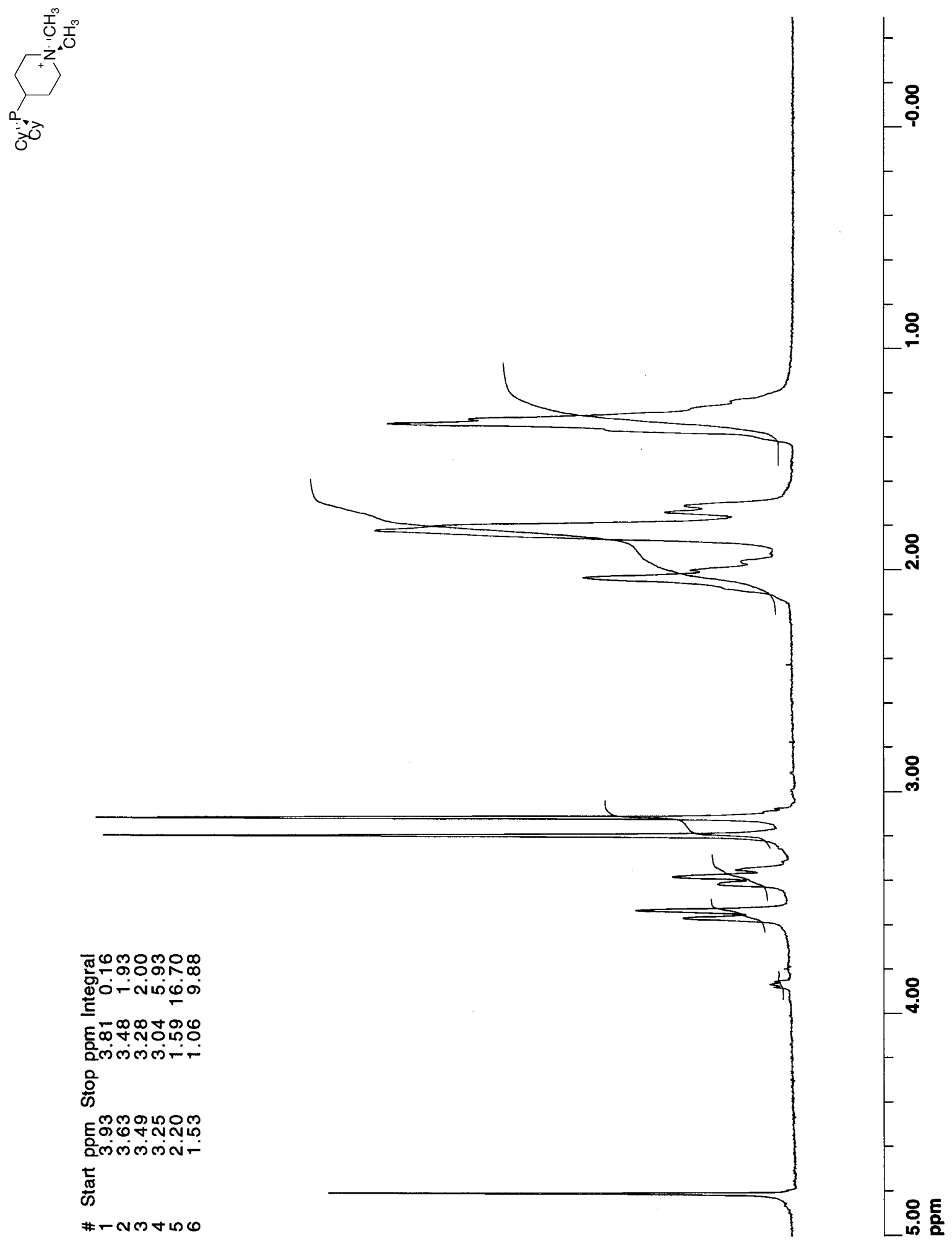

${ }^{1} \mathrm{H}$ NMR spectrum of Cy-Pip-phos ( $\left.\mathrm{D}_{2} \mathrm{O}, 360 \mathrm{MHz}\right)$. 


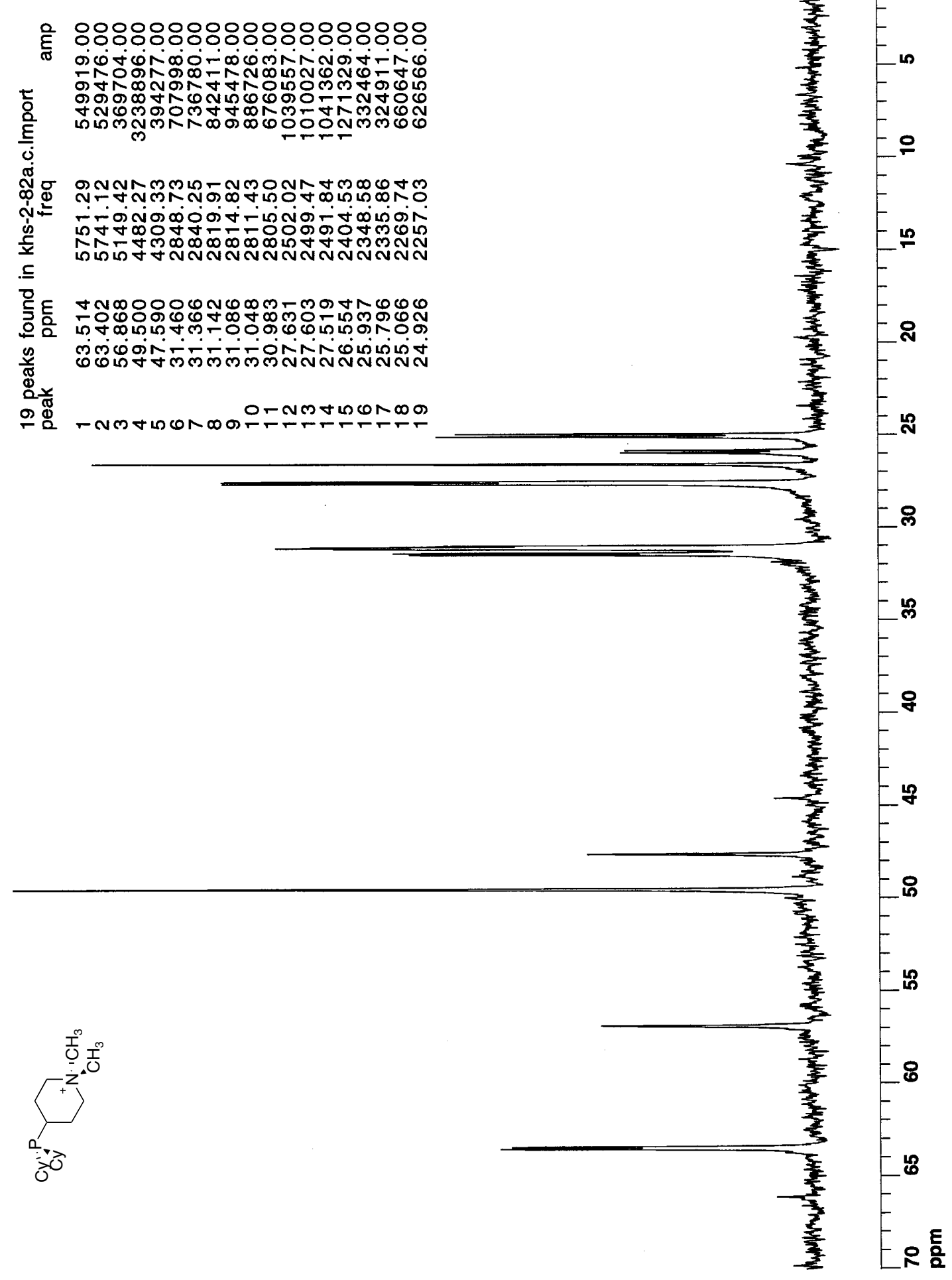

${ }^{13} \mathrm{C}$ NMR spectrum of Cy-Pip-phos $\left(\mathrm{D}_{2} \mathrm{O}, 90.6 \mathrm{MHz}\right)$. Peak at $49.5 \mathrm{ppm}$ is $\mathrm{CH}_{3} \mathrm{OH}$ added as an internal standard. 


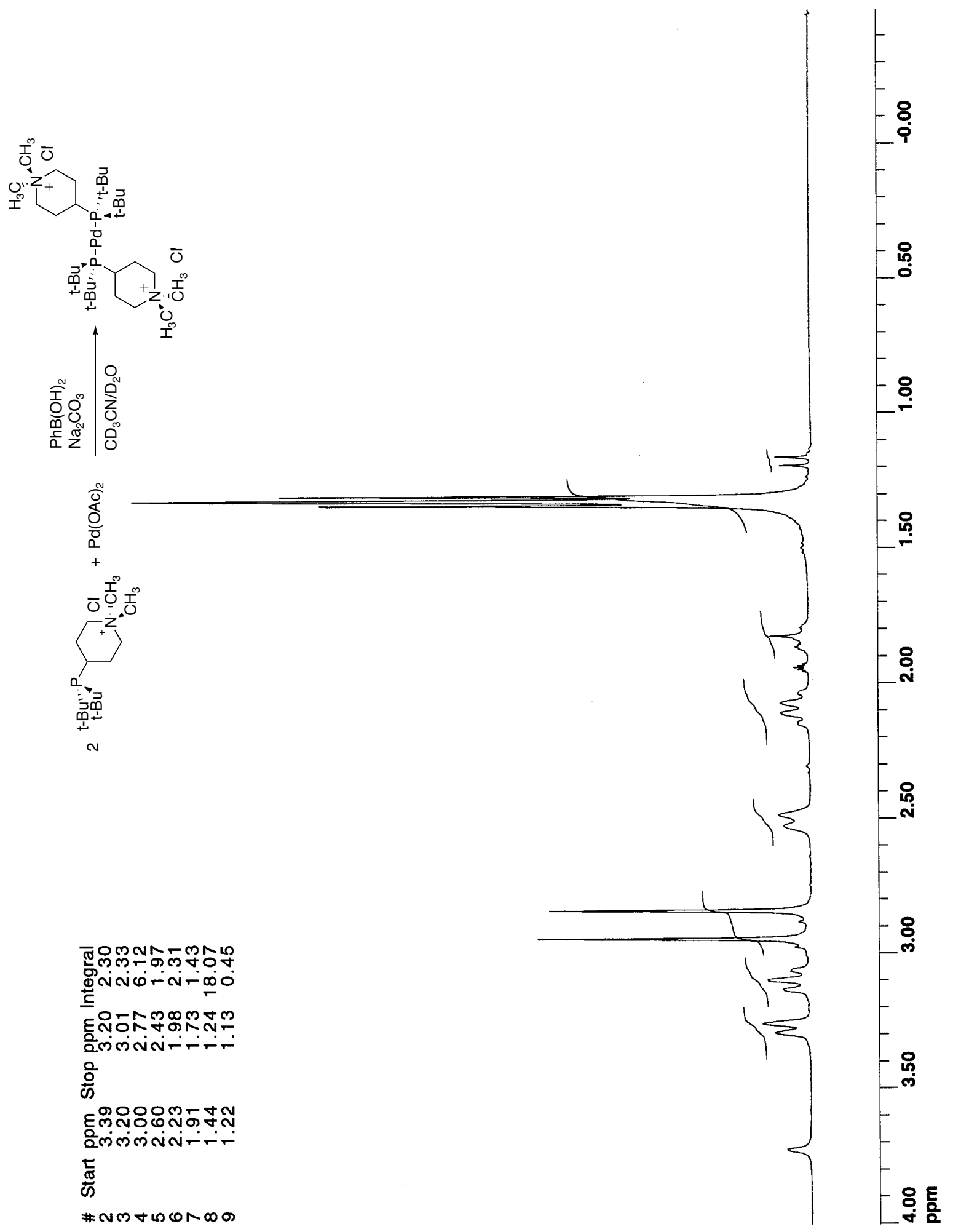

${ }^{1} \mathrm{H}$ NMR spectrum of ( $t$-Bu-Pip-phos $)_{2} \mathrm{Pd}(0)$ generated in situ from $t$-Bu-Pip-phos and $\mathrm{Pd}(\mathrm{OAc})_{2}$ (L:Pd=2:1) in the presence of $\mathrm{PhB}(\mathrm{OH})_{2}$ and $\mathrm{Na}_{2} \mathrm{CO}_{3}\left(\mathrm{CD}_{3} \mathrm{CN} / \mathrm{D}_{2} \mathrm{O}, 360 \mathrm{MHz}\right)$. 


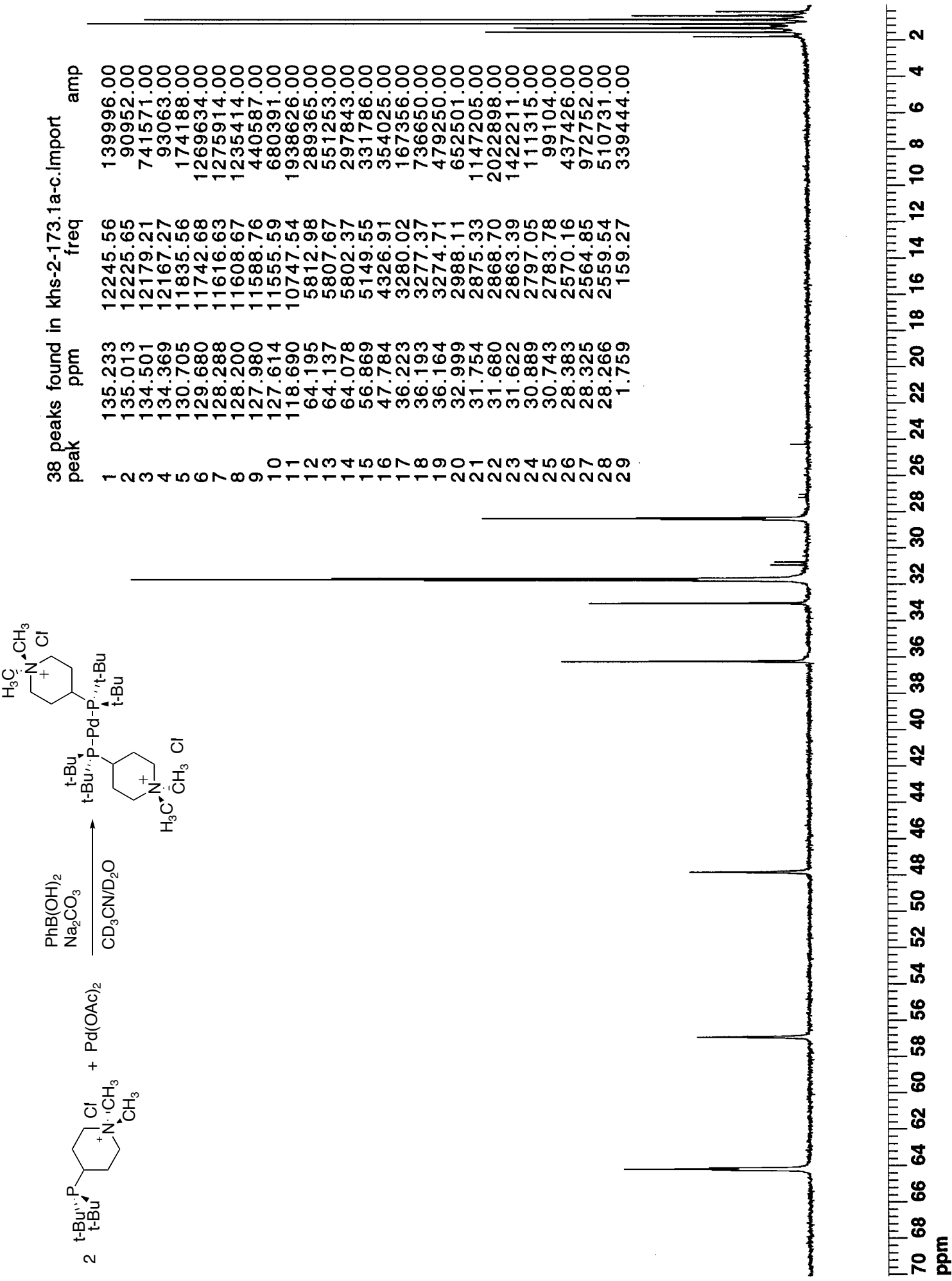

${ }^{13} \mathrm{C}$ NMR spectrum of ( $t$-Bu-Pip-phos $)_{2} \mathrm{Pd}(0)$ generated in situ from $t$-Bu-Pip-phos and $\mathrm{Pd}(\mathrm{OAc})_{2}$ ( $\mathrm{L}: \mathrm{Pd}=2: 1)$ in the presence of $\mathrm{PhB}(\mathrm{OH})_{2}$ and $\mathrm{Na}_{2} \mathrm{CO}_{3}\left(\mathrm{CD}_{3} \mathrm{CN} / \mathrm{D}_{2} \mathrm{O}, 360 \mathrm{MHz}\right)$. 


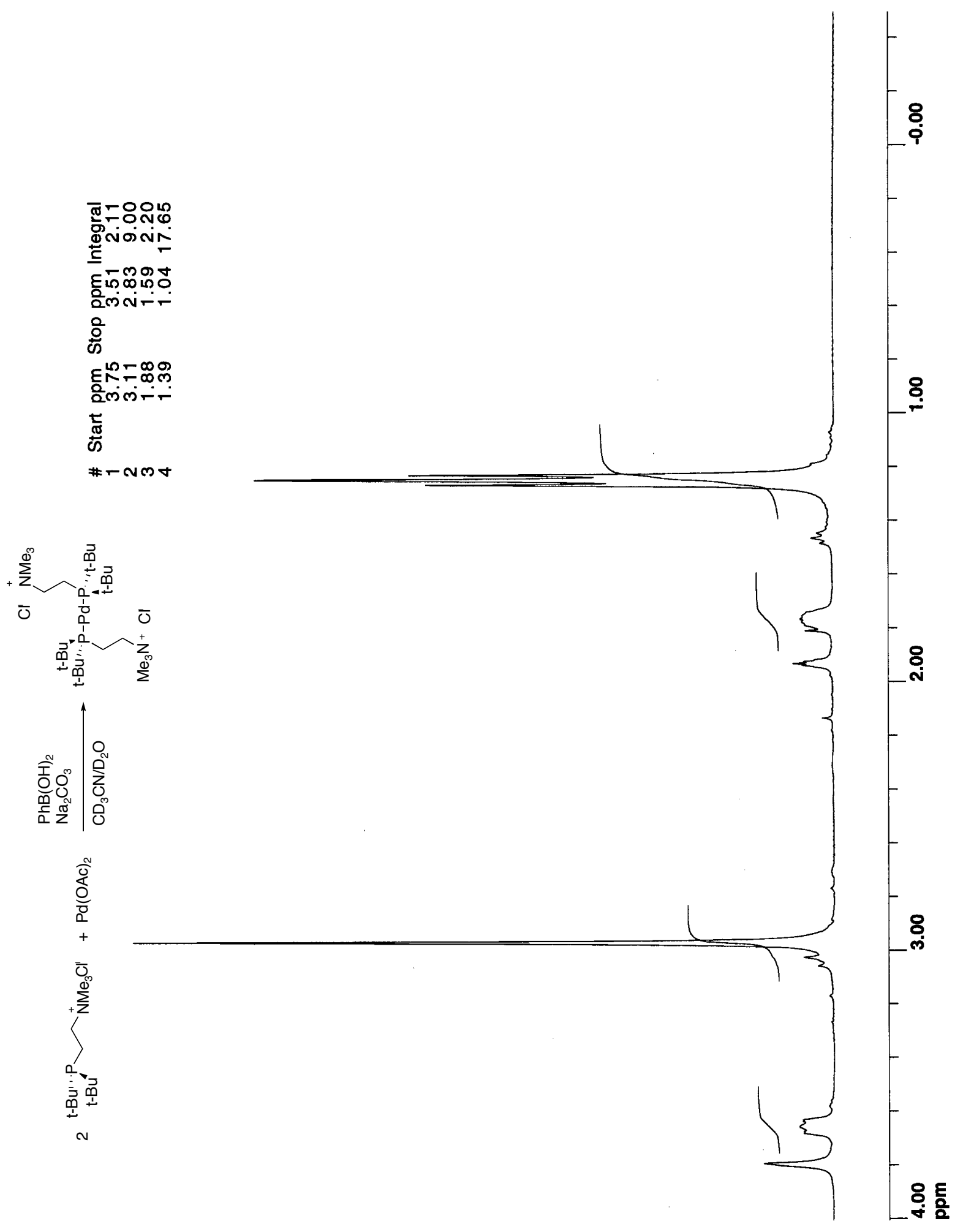

${ }^{1} \mathrm{H}$ NMR spectrum of ( $t$-Bu-Amphos $)_{2} \mathrm{Pd}(0)$ generated in situ from $t$-Bu-Amphos and $\mathrm{Pd}(\mathrm{OAc})_{2}$ ( $\mathrm{L}: \mathrm{Pd}=2: 1)$ in the presence of $\mathrm{PhB}(\mathrm{OH})_{2}$ and $\mathrm{Na}_{2} \mathrm{CO}_{3}\left(\mathrm{CD}_{3} \mathrm{CN} / \mathrm{D}_{2} \mathrm{O}, 360 \mathrm{MHz}\right)$. 


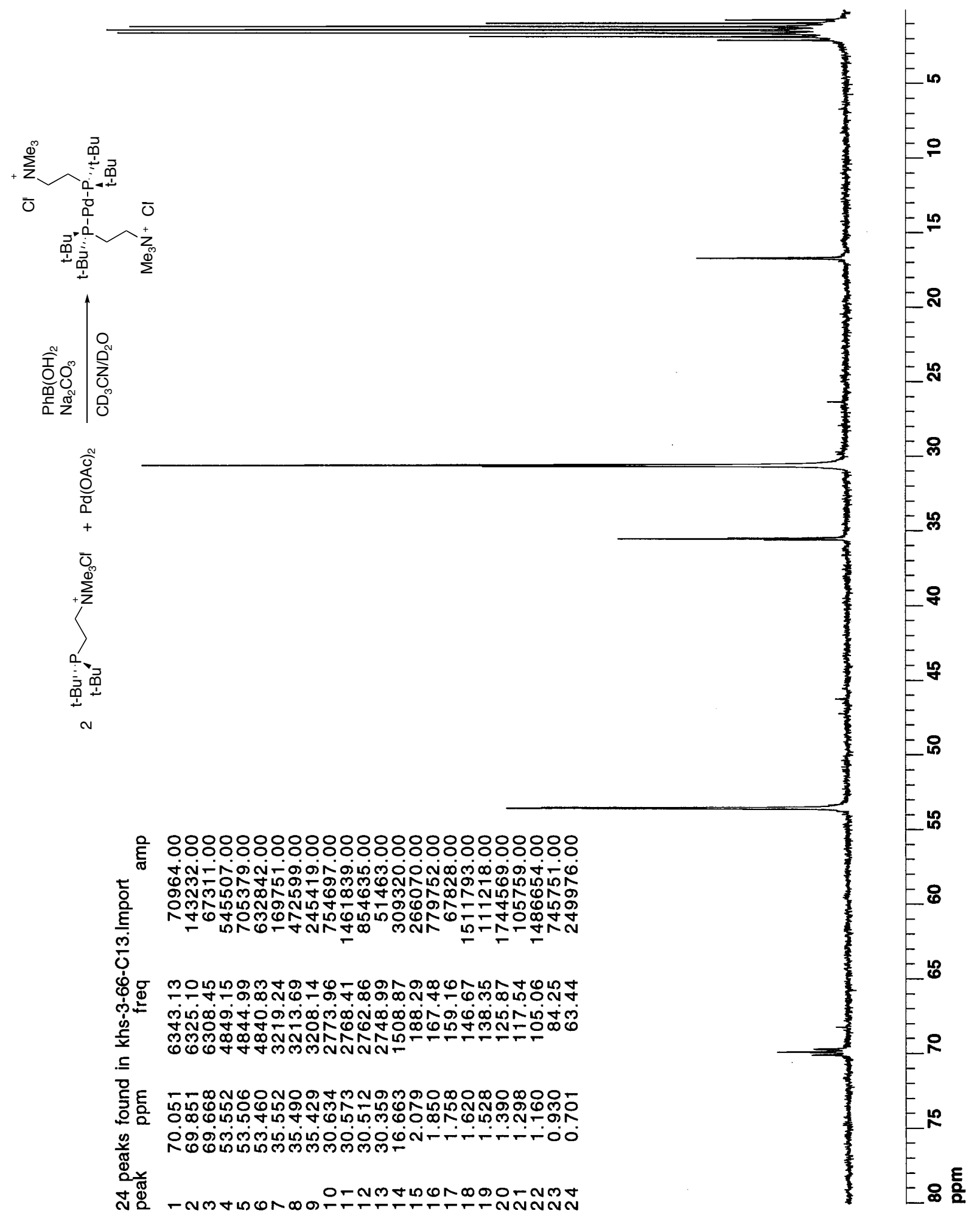

${ }^{13} \mathrm{C}$ NMR spectrum of ( $t$-Bu-Amphos $)_{2} \mathrm{Pd}(0)$ generated in situ from $t$-Bu-Amphos and $\mathrm{Pd}(\mathrm{OAc})_{2}$ $(\mathrm{L}: \mathrm{Pd}=2: 1)$ in the presence of $\mathrm{PhB}(\mathrm{OH})_{2}$ and $\mathrm{Na}_{2} \mathrm{CO}_{3}\left(\mathrm{CD}_{3} \mathrm{CN} / \mathrm{D}_{2} \mathrm{O}, 360 \mathrm{MHz}\right)$. 


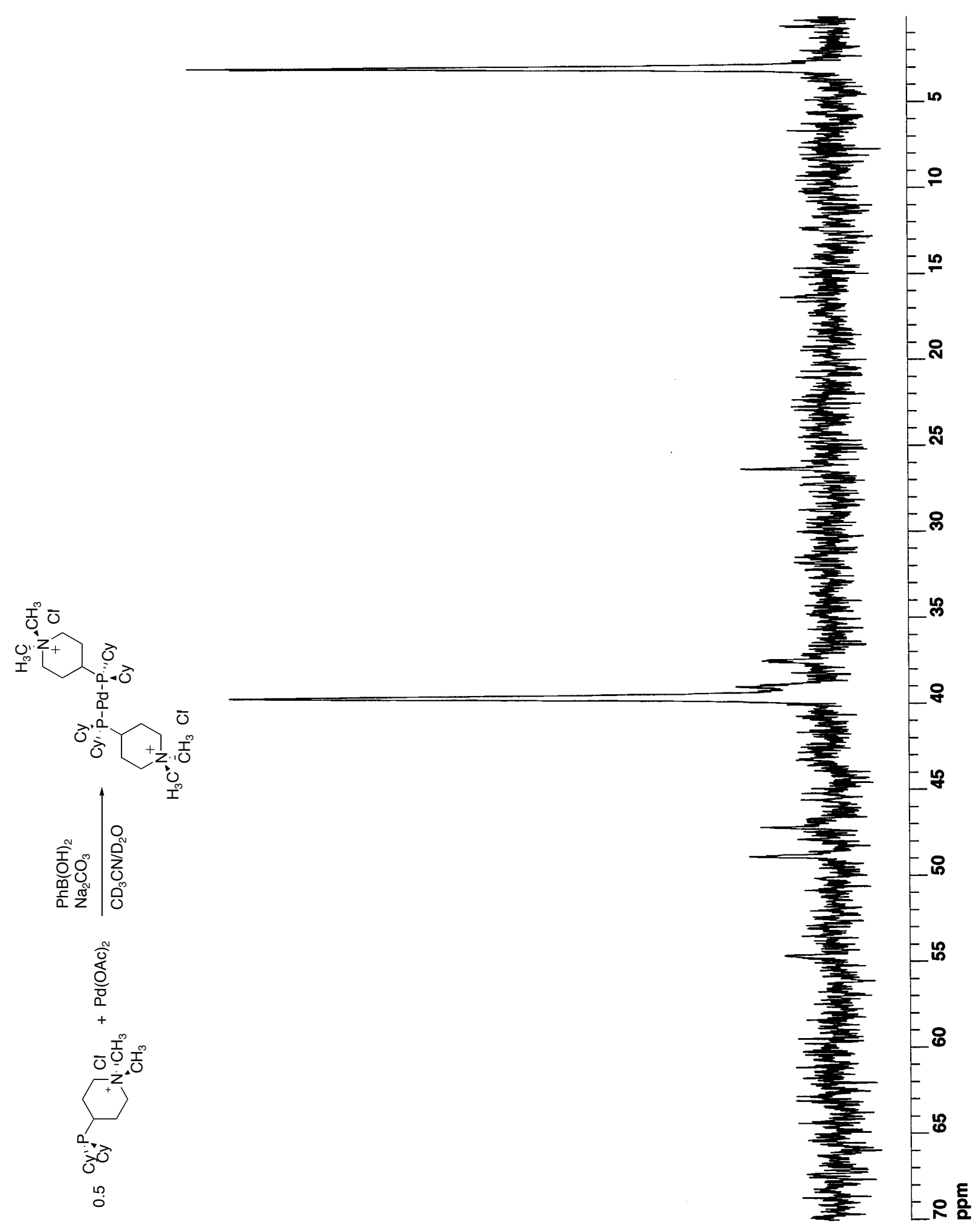

${ }^{13} \mathrm{P}$ NMR spectrum of a 0.5:1 ratio of Cy-Pip-phos: $\mathrm{Pd}(\mathrm{OAc})_{2}$ in the presence of $\mathrm{PhB}(\mathrm{OH})_{2}$ and $\mathrm{Na}_{2} \mathrm{CO}_{3}\left(\mathrm{CD}_{3} \mathrm{CN} / \mathrm{D}_{2} \mathrm{O}, 202.5 \mathrm{MHz}\right)$. Peak at $3 \mathrm{ppm}$ is trimethylphosphate used as an internal standard. 


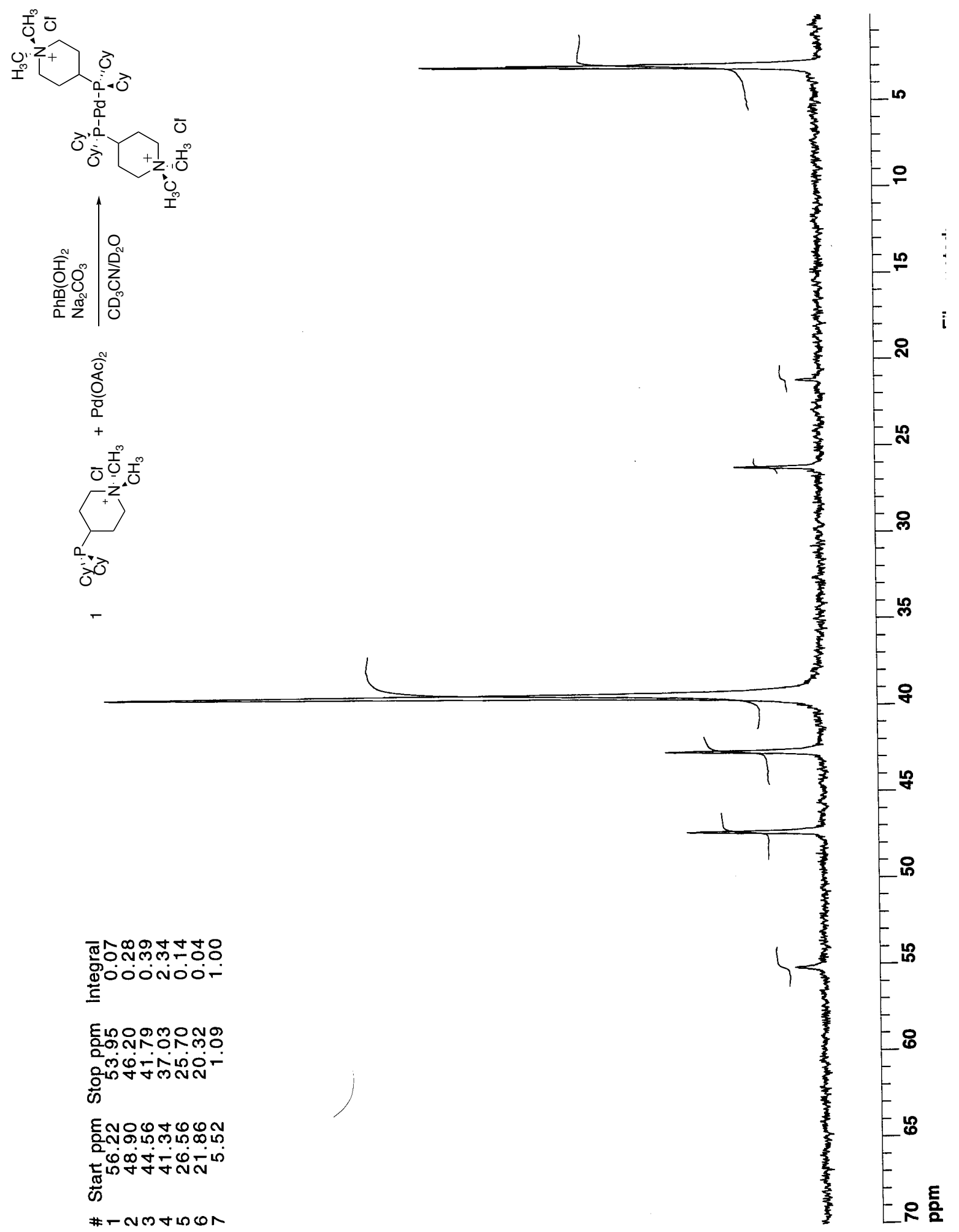

${ }^{13} \mathrm{P}$ NMR spectrum of a 1:1 ratio of Cy-Pip-phos: $\mathrm{Pd}(\mathrm{OAc})_{2}$ in the presence of $\mathrm{PhB}(\mathrm{OH})_{2}$ and $\mathrm{Na}_{2} \mathrm{CO}_{3}\left(\mathrm{CD}_{3} \mathrm{CN} / \mathrm{D}_{2} \mathrm{O}, 202.5 \mathrm{MHz}\right)$. Peak at $3 \mathrm{ppm}$ is trimethylphosphate used as an internal standard. 


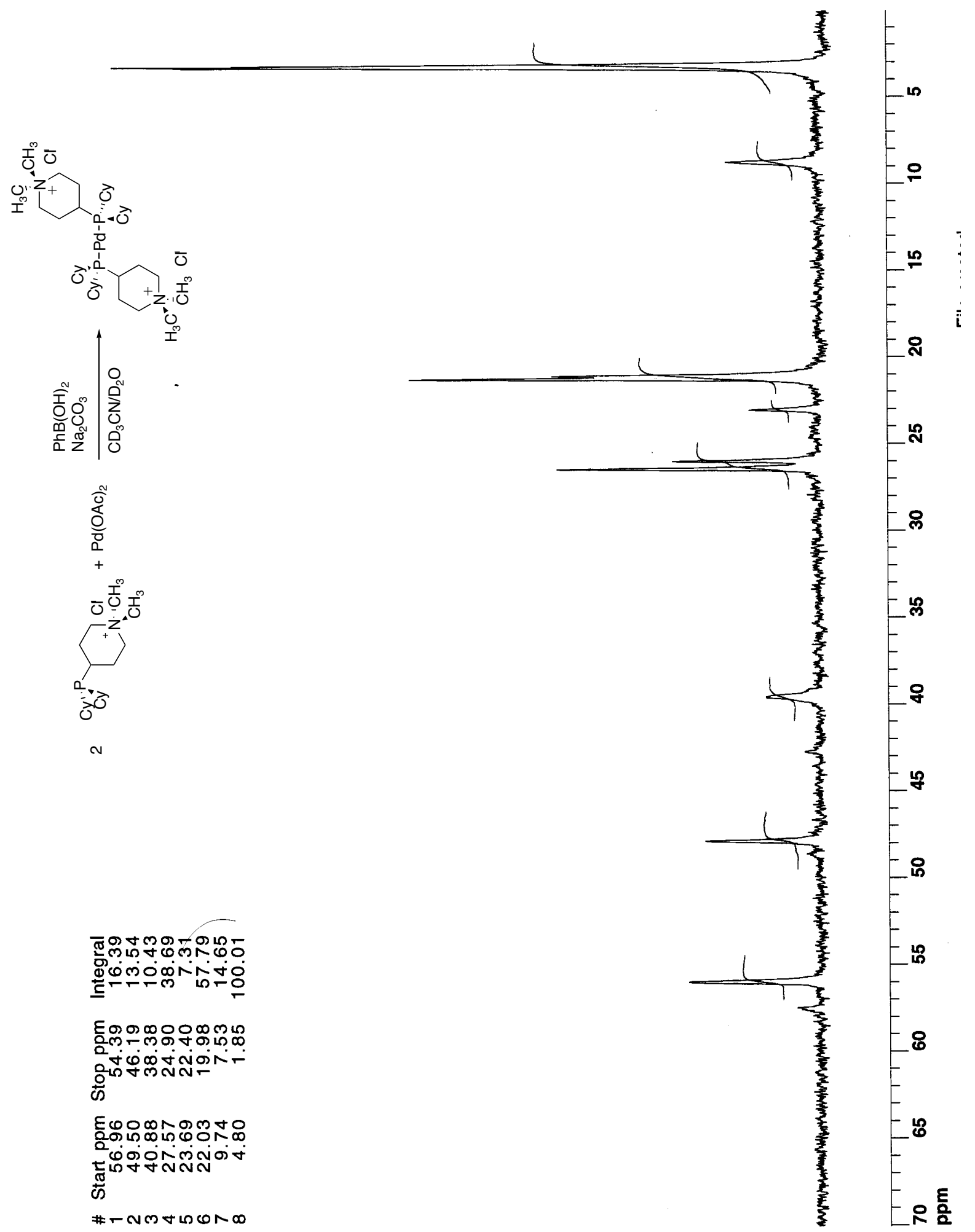

${ }^{13} \mathrm{P}$ NMR spectrum of a 2:1 ratio of Cy-Pip-phos: $\mathrm{Pd}(\mathrm{OAc})_{2}$ in the presence of $\mathrm{PhB}(\mathrm{OH})_{2}$ and $\mathrm{Na}_{2} \mathrm{CO}_{3}\left(\mathrm{CD}_{3} \mathrm{CN} / \mathrm{D}_{2} \mathrm{O}, 202.5 \mathrm{MHz}\right)$. Peak at $3 \mathrm{ppm}$ is trimethylphosphate used as an internal standard. 


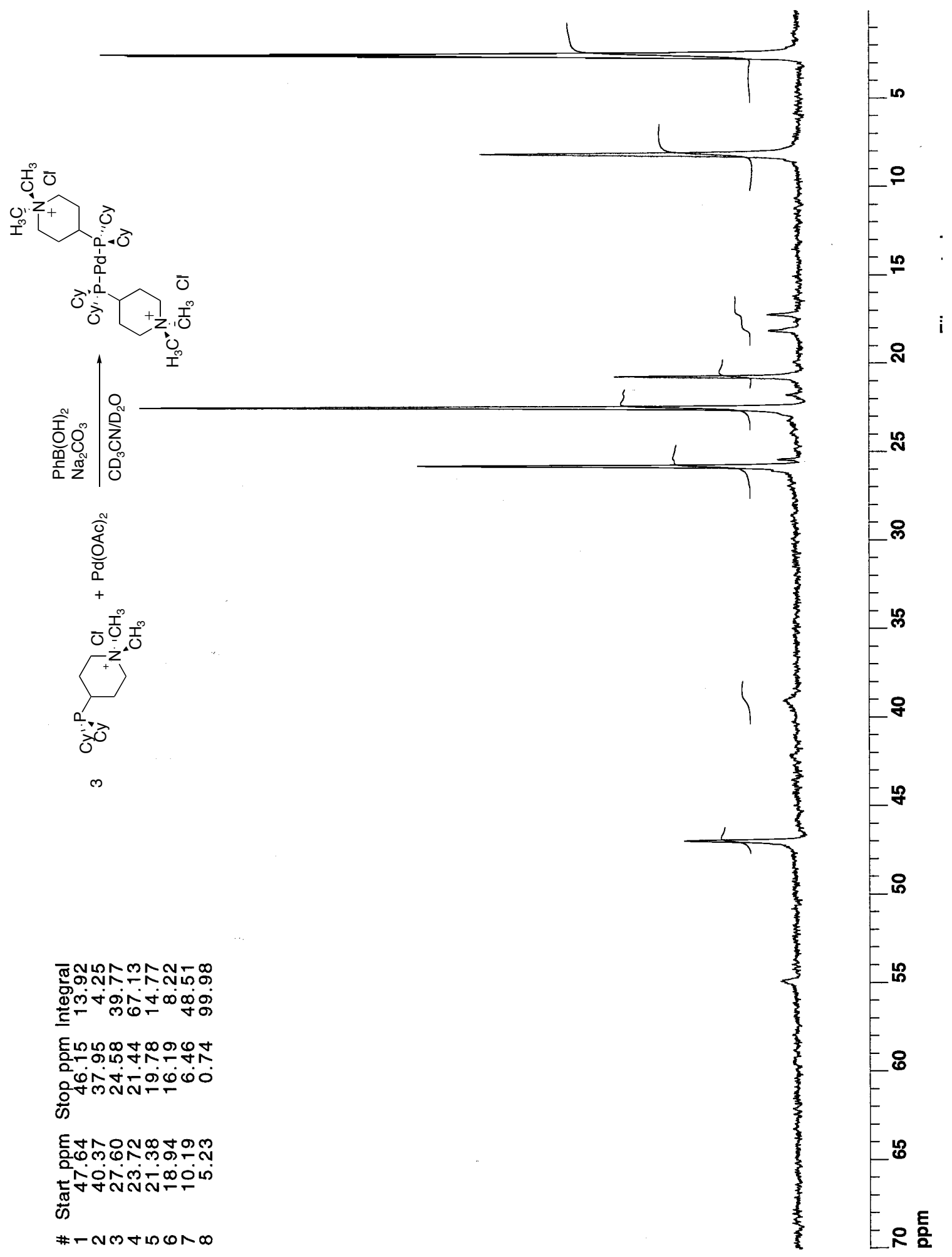

${ }^{13} \mathrm{P}$ NMR spectrum of a 3:1 ratio of Cy-Pip-phos: $\mathrm{Pd}(\mathrm{OAc})_{2}$ in the presence of $\mathrm{PhB}(\mathrm{OH})_{2}$ and $\mathrm{Na}_{2} \mathrm{CO}_{3}\left(\mathrm{CD}_{3} \mathrm{CN} / \mathrm{D}_{2} \mathrm{O}, 202.5 \mathrm{MHz}\right)$. Peak at $3 \mathrm{ppm}$ is trimethylphosphate used as an internal standard. 


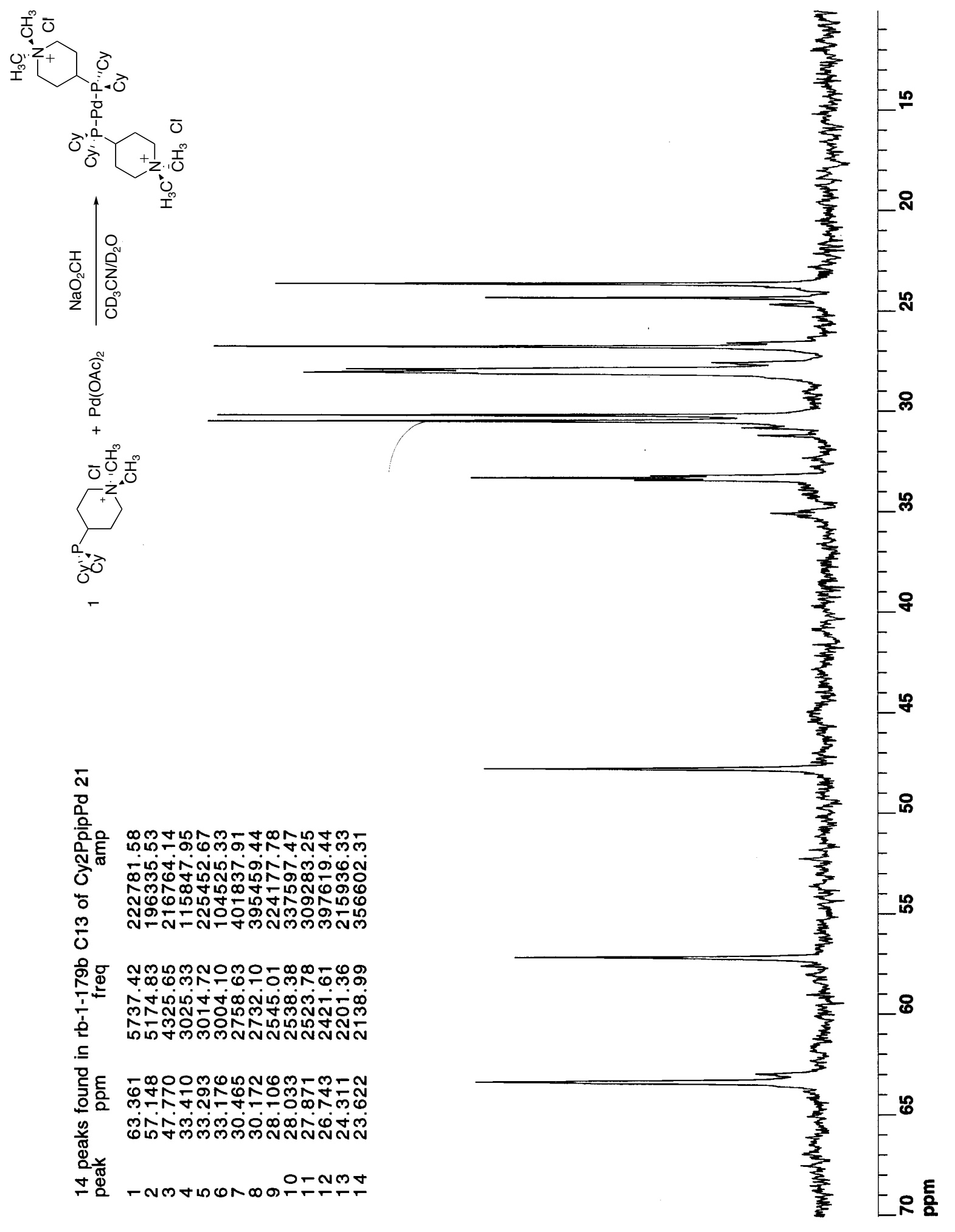

${ }^{13} \mathrm{C}$ NMR spectrum of (Cy-Pip-phos) $)_{2} \mathrm{Pd}(0)$ generated from Cy-Pip-phos and $\mathrm{Pd}(\mathrm{OAc})_{2}(1: 1)$ in the presence of sodium formate $\left(\mathrm{CD}_{3} \mathrm{CN} / \mathrm{D}_{2} \mathrm{O}, 90.6 \mathrm{MHz}\right)$. 


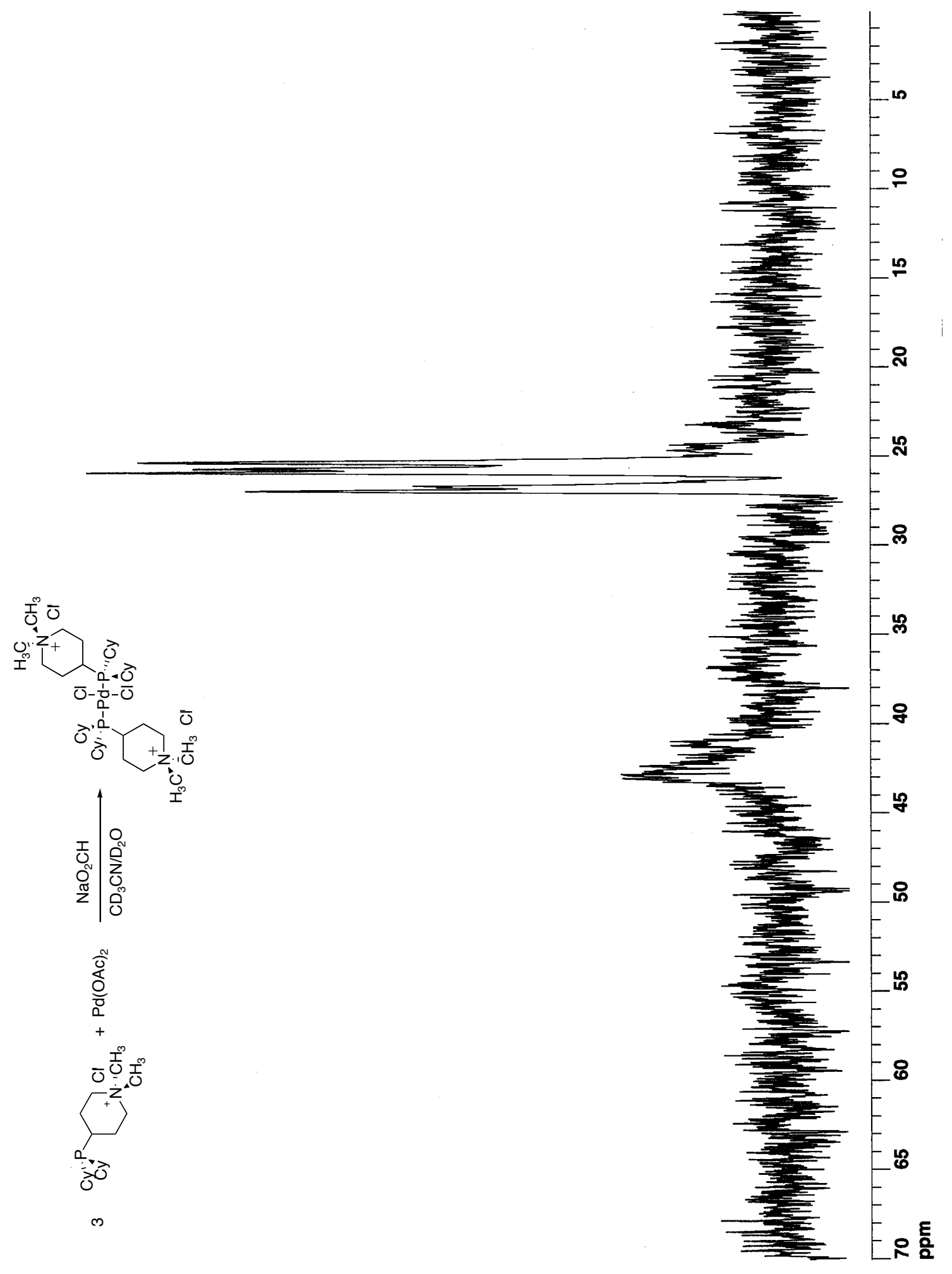

${ }^{31} \mathrm{P}$ NMR spectrum of a 3:1 mixture of Cy-Pip-phos: $\mathrm{Pd}(\mathrm{OAc})_{2}$ in the presence of excess sodium formate after $1 \mathrm{~h}\left(\mathrm{CD}_{3} \mathrm{CN} / \mathrm{D}_{2} \mathrm{O}, 202.5 \mathrm{MHz}\right)$. 


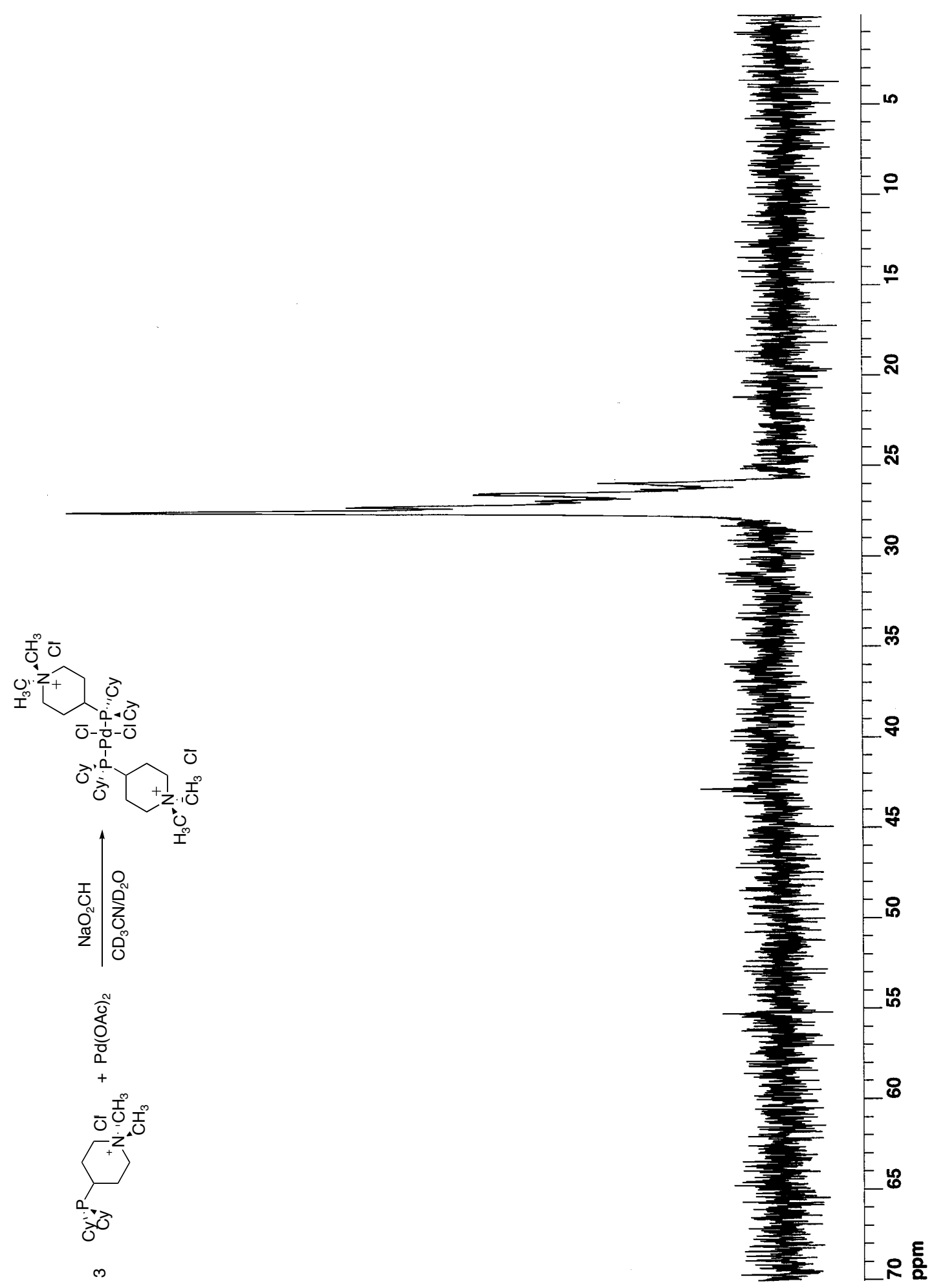

${ }^{31} \mathrm{P}$ NMR spectrum of a 3:1 mixture of Cy-Pip-phos: $\mathrm{Pd}(\mathrm{OAc})_{2}$ in the presence of excess sodium formate after $24 \mathrm{~h}\left(\mathrm{CD}_{3} \mathrm{CN} / \mathrm{D}_{2} \mathrm{O}, 202.5 \mathrm{MHz}\right)$. 


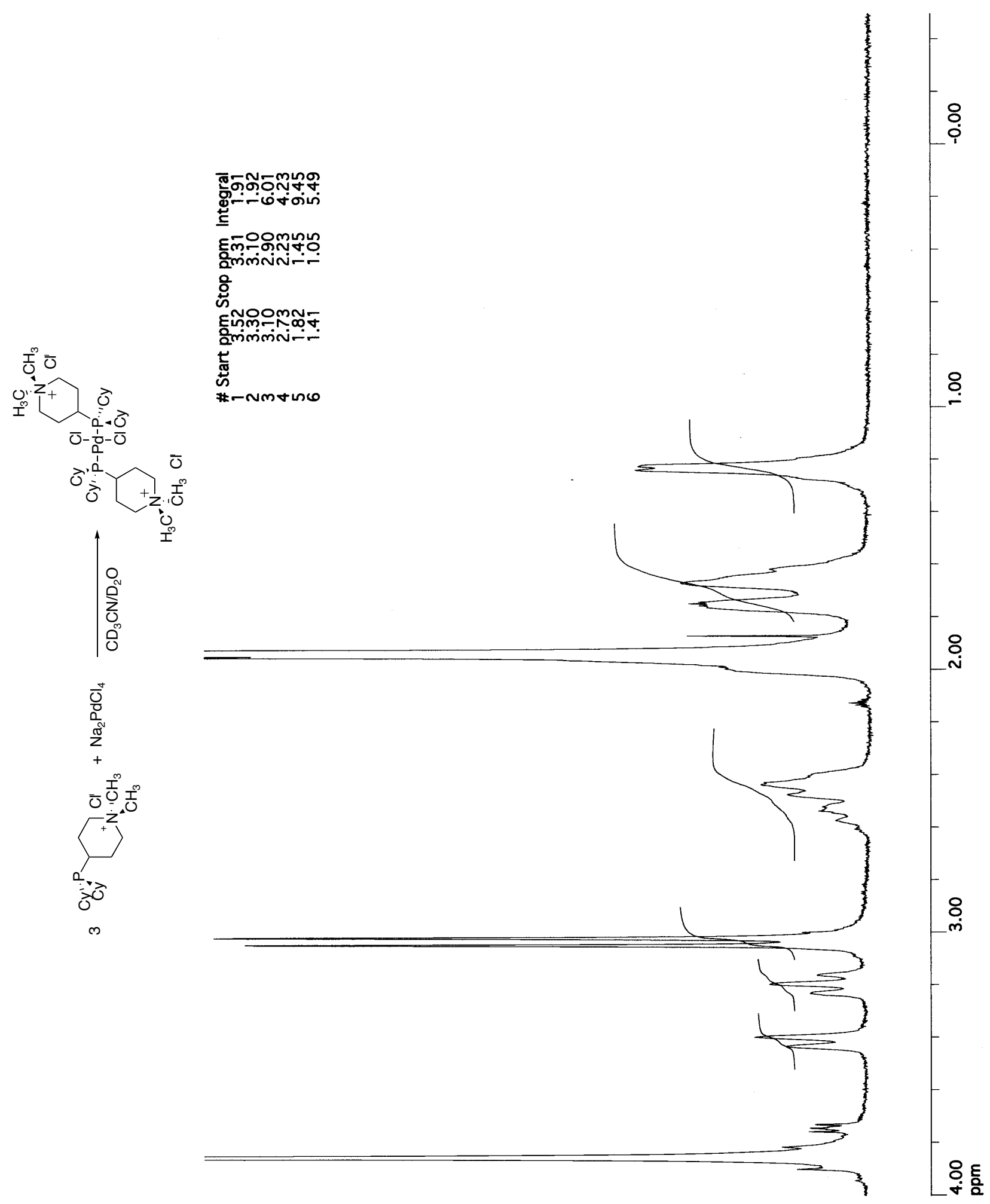

${ }^{13} \mathrm{C}$ NMR spectrum of trans-(Cy-Pip-phos) ${ }_{2} \mathrm{PdCl}_{2}$ generated in situ from Cy-Pip-phos and $\mathrm{Na}_{2} \mathrm{PdCl}_{4}\left(\mathrm{CD}_{3} \mathrm{CN} / \mathrm{D}_{2} \mathrm{O}, 90.6 \mathrm{MHz}\right)$ 


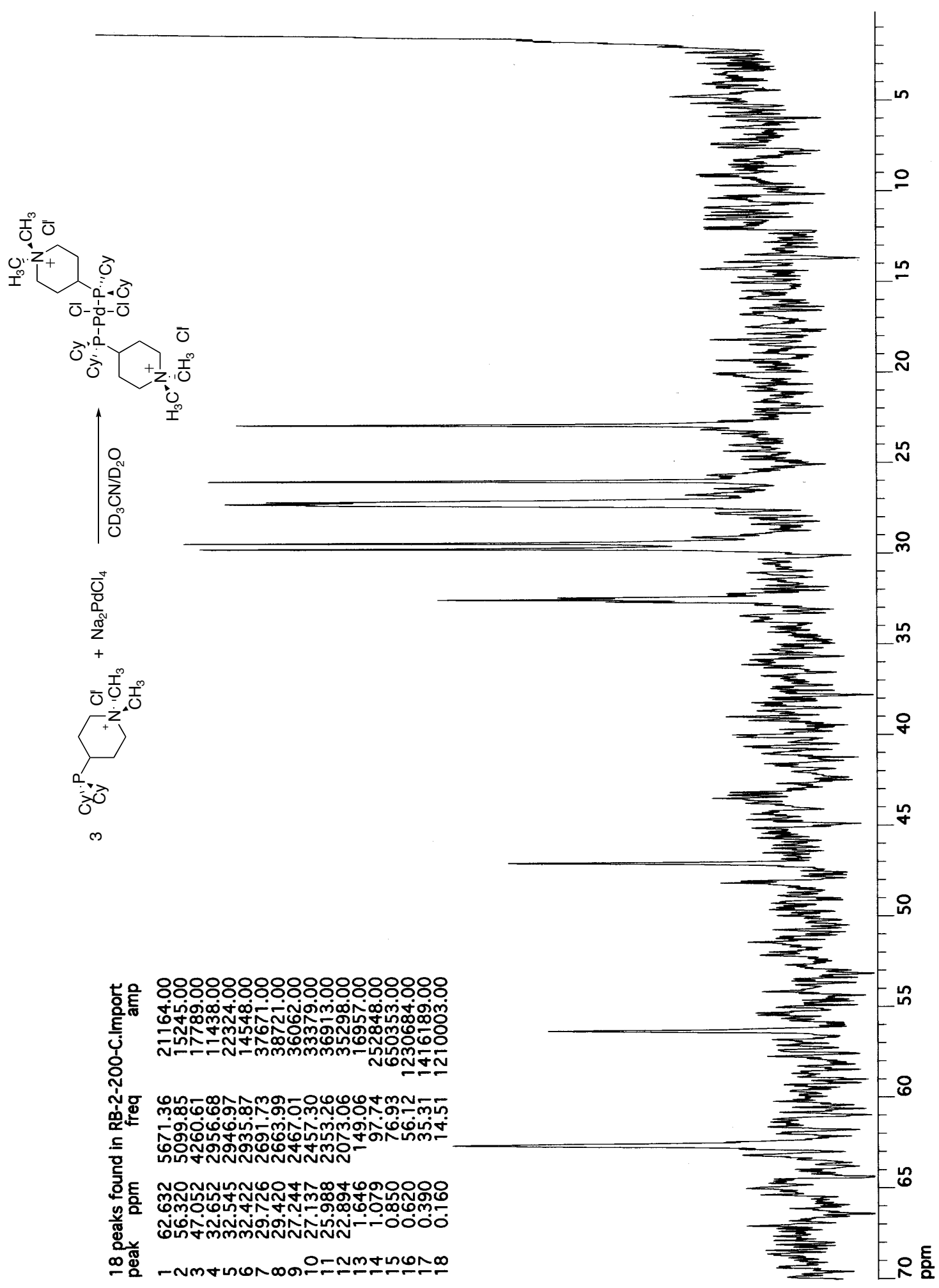

${ }^{13} \mathrm{C}$ NMR spectrum of trans-(Cy-Pip-phos $)_{2} \mathrm{PdCl}_{2}$ generated in situ from Cy-Pip-phos and $\mathrm{Na}_{2} \mathrm{PdCl}_{4}\left(\mathrm{CD}_{3} \mathrm{CN} / \mathrm{D}_{2} \mathrm{O}, 90.6 \mathrm{MHz}\right)$ 

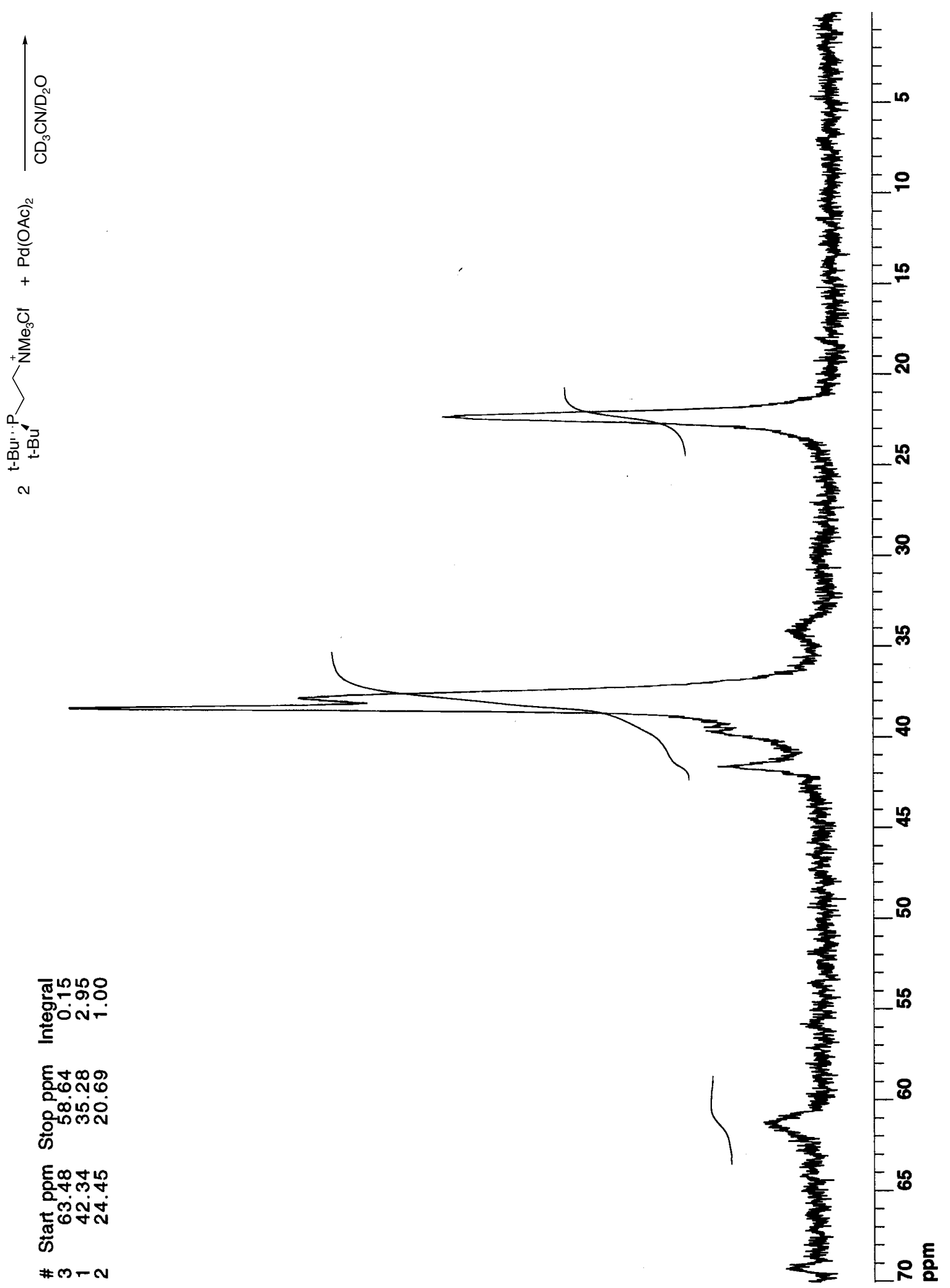

${ }^{31} \mathrm{P}$ NMR spectrum of the reaction of $t$-Bu-Amphos $+\mathrm{Pd}(\mathrm{OAc})_{2}(\mathrm{~L}: \mathrm{Pd}=2: 1)\left(\mathrm{D}_{2} \mathrm{O}, 202.5 \mathrm{MHz}\right)$. 


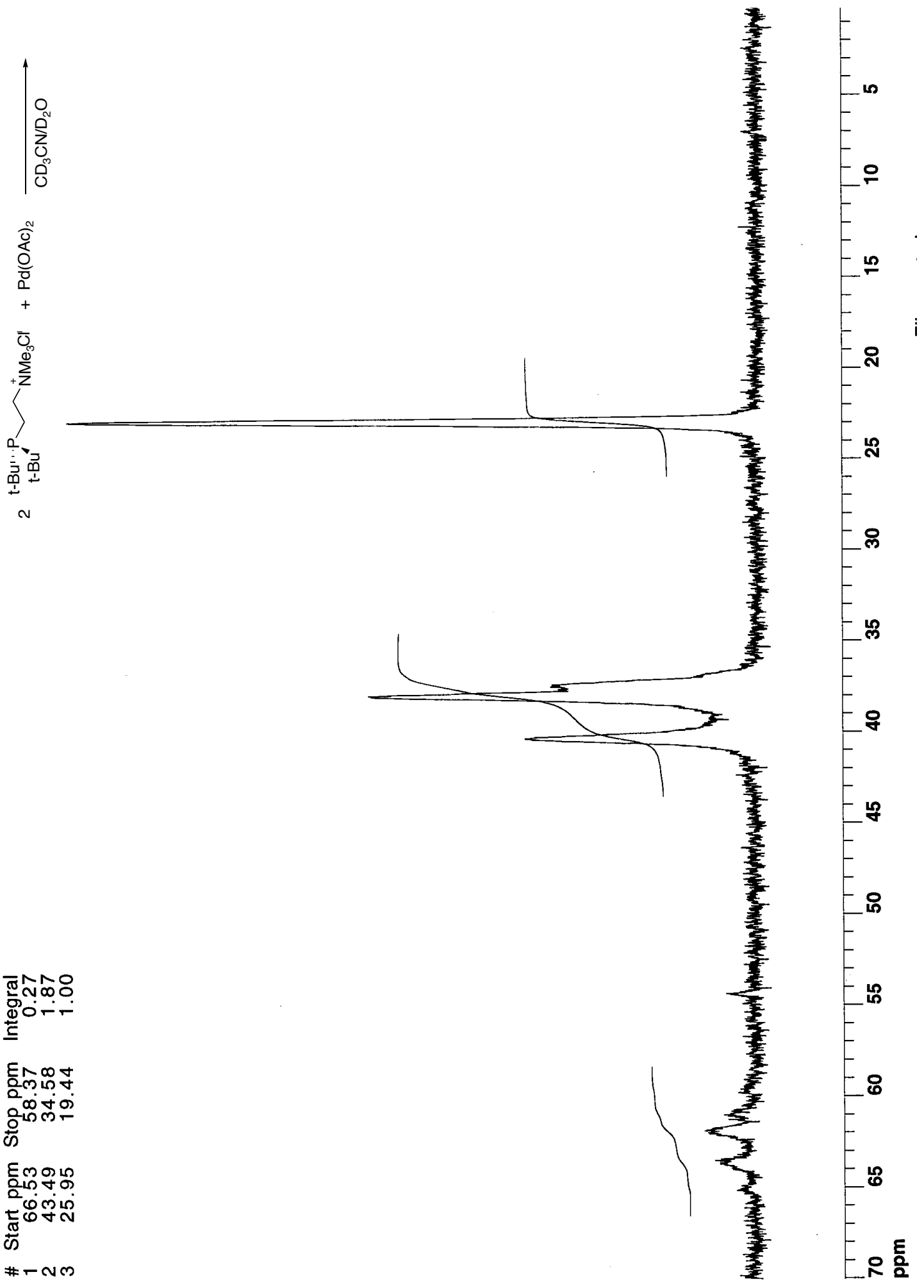

${ }^{31} \mathrm{P}$ NMR spectrum of precipitate formed in reaction of $t$-Bu-Amphos $+\mathrm{Pd}(\mathrm{OAc})_{2}\left(\mathrm{CH}_{3} \mathrm{CN}, 202.5\right.$ $\mathrm{MHz}$ ). 